\title{
Complex organics in space from Solar System to distant galaxies
}

\author{
Sun Kwok ${ }^{1}$
}

Received: 12 November 2015 / Published online: 29 February 2016

(C) The Author(s) 2016. This article is published with open access at Springerlink.com

\begin{abstract}
Recent observational and experimental evidence for the presence of complex organics in space is reviewed. Remote astronomical observations have detected $\sim 200$ gas-phased molecules through their rotational and vibrational transitions. Many classes of organic molecules are represented in this list, including some precursors to biological molecules. A number of unidentified spectral phenomena observed in the interstellar medium are likely to have originated from complex organics. The observations of these features in distant galaxies suggests that organic synthesis had already taken place during the early epochs of the Universe. In the Solar System, almost all biologically relevant molecules can be found in the soluble component of carbonaceous meteorites. Complex organics of mixed aromatic and aliphatic structures are present in the insoluble component of meteorites. Hydrocarbons cover much of the surface of the planetary satellite Titan and complex organics are found in comets and interplanetary dust particles. The possibility that the early Solar System, or even the early Earth, have been enriched by interstellar organics is discussed.
\end{abstract}

Keywords Astrochemistry · Astrobiology · Interstellar molecules

\section{Introduction}

Before the nineteenth century, organic matter was assumed to be associated only with life and was believed to possess a special quality called the "vital force". This belief was based on the fact that biologically related molecules were all derived from

Sun Kwok

sunkwok@hku.hk

1 Laboratory for Space Research, Faculty of Science, The University of Hong Kong,

Hong Kong, China 
living organisms. For example, the amino acid asparagine was derived from asparagus, leucine from cheese, glycine from gelatin, and living yeast was needed for fermentation of sugar into alcohol. However, advances in laboratory chemical techniques have made possible the artificial synthesis of urea $\left(\mathrm{CO}\left(\mathrm{NH}_{2}\right)_{2}\right)$ from ammonium cyanate $\left(\mathrm{NH}_{4} \mathrm{CNO}\right)$ in 1823, amino acid alanine from acetaldehyde, ammonia, and hydrogen cyanide in 1850 , and sugars from formaldehyde in 1861 . The ability to synthesize organic matter from inorganic ingredients has led to the demise of the "vital force" concept. We now define organics as the class of molecules and compounds based on the element carbon, but they contain also other elements such as $\mathrm{H}, \mathrm{O}, \mathrm{N}, \mathrm{S}$, and $\mathrm{P}$.

On Earth, organic matter as the result of life is present in the oceans, in the atmosphere, and on the Earth's crust. However, the total amount of biomass ( $\sim 2000$ giga tons) pales in comparison to the amount of kerogen, $\left(>1.5 \times 10^{7}\right.$ giga tons), a complex, high molecular weight organic matter found in sedimentary rocks (Falkowski et al. 2000). Kerogen is believed to be a precursor of fossil fuels such as oil, coal, and natural gas and therefore represents remnants of past life on Earth (Dow 1977).

Until very recently, complex organics was thought to be in the exclusive domain of the Earth. The detection of a large number of gas-phase organic molecules in the interstellar medium through their rotational and vibrational transitions gave us the first hint that organic matter can be naturally synthesized in space. Solid-state particles (commonly referred to in the astronomical literature as dust or grains) have been known to exist in the interstellar medium through the technique of selective extinction for about 100 years. Early suggestions of the chemical structure of interstellar solids included graphite, iron, and ice. The detection of the $10 \mu \mathrm{m}$ silicate feature in evolved stars and in the interstellar medium in the late 1960s led to the realization that minerals such as amorphous silicates are a major component of interstellar solids (Woolf and Ney 1969). Although the existence of organic solids in space has been speculated for some time (Hoyle and Wickramasinghe 1977; Knacke 1977), this idea has never been taken seriously by the astronomical community until recently. There is now strong evidence that complex organic matter is widely present in the Solar System, in the circumstellar environment of stars, in interstellar clouds, in the diffuse interstellar medium, and in distant galaxies. In this paper, I will review our current observational evidence for complex organics in various astronomical environments and discuss the chemical structure and origins of organic solids in space.

\section{Organic molecules in the gas phase}

The first interstellar molecules detected were $\mathrm{CN}, \mathrm{CH}$, and $\mathrm{CH}^{+}$through their electronic transition in absorption against background starlight (McKellar 1940). The possibility of detecting molecular transitions in the microwave part of the electromagnetic spectrum was recognized by Shklovsky and Townes in the early 1950s (Townes 1957). In spite of this knowledge, the general feeling in the astronomical community was that it is unlikely for molecules other than simple radicals to exist in space because of the low gas density and high ultraviolet radiation background (see, e.g., Townes 2006). Search for molecular transitions was made possible by technical advances in microwave, millimeter-wave and infrared detectors in the 1960s. 
Since the first detection of simple molecules such as $\mathrm{OH}$ (detected in 1963), $\mathrm{NH}_{3}$ (1968), $\mathrm{H}_{2} \mathrm{O}$ (1969), CO (1970), approximately 200 gas-phase molecules have been detected in the interstellar medium, including over 80 in the circumstellar envelopes of late-type stars (http://www.astro.uni-koeln.de/cdms/molecules). The detected species cover all kinds of organic molecules, including hydrocarbons (e.g., methane $\mathrm{CH}_{4}$, acetylene $\mathrm{C}_{2} \mathrm{H}_{2}$, ethylene $\mathrm{C}_{2} \mathrm{H}_{4}$ ), alcohols (e.g., methanol $\mathrm{CH}_{3} \mathrm{OH}$, ethanol $\mathrm{C}_{2} \mathrm{H}_{5} \mathrm{OH}$, vinyl alcohol $\mathrm{H}_{2} \mathrm{C}=\mathrm{CHOH}$ ), acids (e.g., formic acid $\mathrm{HCOOH}$, acetic acid $\mathrm{CH}_{3} \mathrm{COOH}$ ), aldehydes (e.g., formaldehyde $\mathrm{H}_{2} \mathrm{CO}$, acetaldehyde $\mathrm{CH}_{3} \mathrm{CHO}$, propenal $\mathrm{CH}_{2}=\mathrm{CHCHO}$, propanal $\mathrm{CH}_{3} \mathrm{CH}_{2} \mathrm{CHO}$ ), ketones (e.g., ethenone $\mathrm{H}_{2} \mathrm{C}=\mathrm{CO}$, acetone, $\mathrm{CH}_{3} \mathrm{COCH}_{3}$ ), amines (e.g., methylamine $\mathrm{CH}_{3} \mathrm{NH}_{2}$, cyanamide $\mathrm{NH}_{2} \mathrm{CN}$, formamide $\mathrm{NH}_{2} \mathrm{CHO}$ ), ethers (e.g., dimethyl ether $\mathrm{CH}_{3} \mathrm{OCH}_{3}$, ethyl methyl ether $\mathrm{CH}_{3} \mathrm{OC}_{2} \mathrm{H}_{5}$ ), etc.

Large asymmetric organic molecules have complicated energy structures and correspondingly large partition functions. Their populations are spread over many energy states, making individual rotational transitions weak (Kwok 2006). A firm detection of complex gas-phase organic molecules requires the matching of multiple transitions. Some simple asymmetric molecules such as methanol $\left(\mathrm{CH}_{3} \mathrm{OH}\right)$, and to a less extent dimethyl ether $\left(\mathrm{CH}_{3} \mathrm{OCH}_{3}\right)$, vinyl cyanide $\left(\mathrm{CH}_{2} \mathrm{CHCN}\right)$, ethanol $\left(\mathrm{C}_{2} \mathrm{H}_{5} \mathrm{OH}\right)$, and methyl formate $\left(\mathrm{HCOOCH}_{3}\right)$, are so abundant and have so many transitions that their lines crowd out the weaker lines of more complex molecules. The sensitivities of modern millimeter $(\mathrm{mm})$ and submm-wave telescopes are so high and the quantity of interstellar molecules so abundant that practically every spectral region of the $\mathrm{mm} / \mathrm{submm}$ spectrum is filled with some molecular line. This is evident in spectral line surveys carried out by modern telescopes such as the Herschel Space Telescope (Neill et al. 2014; Crockett et al. 2014). This phenomenon, known as line confusion, greatly limits our ability to search for weaker lines. The failure to detect complex organic molecules through their rotational transitions therefore does not necessarily imply the absence or low abundance of the molecules.

The classes of carbohydrates, lipids, proteins, and nucleic acids form the four basic classes of organic molecules of life. One of the major recent efforts in astrochemistry has been to search for the building blocks of these molecules. In this review, we will concentrate on the study of complex organics. A review of simpler gas-phase organic molecules, in particular those in dense molecular clouds, can be found in Caselli and Ceccarelli (2012). Below, we will summarize some of the progress made in the search for complex organics in the Universe.

\subsection{Amines and amides}

The element nitrogen $(\mathrm{N})$ plays an important role in biochemistry as it is needed in the synthesis of amino acids and nucleotides. Hydrogen cyanide (HCN), a precursor of many biomolecules, and the family of cyanopolynnes $\left(\mathrm{HC}_{n} \mathrm{~N}\right)$ are easily detectable through their rotational transitions because of the linear structure of the molecules and their degenerate rotational states. The wide presence of $\mathrm{N}$-containing molecules in the interstellar medium provides the confidence needed that the $\mathrm{N}$ is indeed available for biochemical synthesis in space. 
The classes of $\mathrm{N}$-containing molecules amines and amides therefore could serve as first building blocks of biomolecules. The simplest naturally occurring amide is formamide $\left(\mathrm{NH}_{2} \mathrm{CHO}\right)$, which was detected as early as 1971 (Rubin et al. 1971). Other examples include cyanamide $\left(\mathrm{H}_{2} \mathrm{NCN}\right.$, Turner et al. 1975), acetamide $\left(\mathrm{H}_{2} \mathrm{NCOCH}_{3}\right.$ Hollis et al. 2006), and methylamine $\left(\mathrm{H}_{3} \mathrm{CNH}_{2}\right.$, Fourikis et al. 1974; Kaifu et al. 1974).

Urea $\left(\left(\mathrm{H}_{2} \mathrm{~N}\right)_{2} \mathrm{CO}\right)$ was the first biomolecule synthesized in the laboratory, demonstrating the ingredients of life can be artificially manufactured. The detection of urea in space would therefore possess special significance. Although the rotational spectra of urea is well known, the spectral complexity of the molecules makes definite detection of this molecule difficult (Remijan et al. 2014).

\subsection{Sugars}

Sugars (monosaccharides) are a class of carbohydrates. The simplest sugar is glyceraldehyde $\left(\mathrm{CH}_{2} \mathrm{OHCHOHCHO}\right)$. Although glyceraldehyde has been searched but not found (Hollis et al. 2004), a simpler related molecule glycolaldehyde $\left(\mathrm{CH}_{2} \mathrm{OHCHO}\right.$, Carroll et al. 2010) has been detected (Hollis et al. 2000; Jørgensen et al. 2012).

\subsection{Amino acids and proteins}

There have been extensive searches for glycine, the simplest amino acid (Kuan et al. 2003a, b; Snyder et al. 2005), but so far there has been no concrete evidence for its detection (Cunningham et al. 2007; Jones et al. 2007). Glycine in the interstellar medium is assumed to be formed on ice under UV irradiation (Kim and Kaiser 2011). The immediate precursors of gylcine are $\mathrm{CH}_{3} \mathrm{NH}_{2}$ (formed with $\mathrm{CO}_{2}$ ) or $\mathrm{CH}_{2} \mathrm{NH}_{2}$ (formed with $\mathrm{HCN}$ ), both of which have been detected (Kaifu et al. 1974; Ohishi 2015). Amino acetonitrile $\left(\mathrm{NH}_{2} \mathrm{CH}_{2} \mathrm{CN}\right)$, a molecule chemically related to glycine although not necessarily its precursor, has also been detected (Belloche et al. 2008).

Proteins (polypeptides) are polymers of amino acids joined by peptide $(-\mathrm{NH}-(=\mathrm{O})-$ )) bonds. Examples of molecules with peptide bonds detected in the interstellar medium are formamide ( $\mathrm{NH}_{2} \mathrm{CHO}$ ) (Rubin et al. 1971; Kahane et al. 2013; Mendoza et al. 2014) and acetamide $\left(\mathrm{CH}_{3} \mathrm{CONH}_{2}\right)($ Hollis et al. 2006; Halfen et al. 2011).

\subsection{Heterocyclic molecules}

Planar rings with $\mathrm{N}$, O, or $\mathrm{S}$ replacing one of the $\mathrm{C}$ atoms on the ring are important building blocks of biomolecules. Examples of precursors to heterocyclic molecules include furan $\left(\mathrm{C}_{4} \mathrm{H}_{4} \mathrm{O}\right)$, pyrrole $\left(\mathrm{C}_{4} \mathrm{H}_{5} \mathrm{~N}\right)$, and imidazole $\left(\mathrm{C}_{3} \mathrm{H}_{4} \mathrm{~N}_{2}\right)$. Pyrrole serves as a precursor for the side train of the amino acid proline and is a constituent of the heme groups in hemoglobin and chlorophyll. Imidazole is formed as a side chain in the amino acid histidine and the biomolecule histamine. The monocyclic pyrimidine $\left(c-\mathrm{C}_{4} \mathrm{H}_{4} \mathrm{~N}_{2}\right)$ and bicyclic purine $\left(c-\mathrm{C}_{5} \mathrm{H}_{4} \mathrm{~N}_{4}\right)$ molecules are the parents of the nucleobases. Pyrimidine is the base for cytosine (DNA and RNA), thymine (DNA), and 
uracil (RNA), whereas purine is the basis for adenine and guanine (in both DNA and RNA).

The structures of these molecules are simple enough that it is possible to search for them via their rotational transitions. However, searches to date have yielded only negative results [pyrrole: Kutner et al. (1980), furan: Dickens et al. (2001), imidazole: Irvine et al. (1981), pyrimidine: Charnley et al. (2005)].

\section{Organics in the Solar System}

While astronomical sources are only accessible by remote passive observations, Solar System objects have the advantage that physical samples can be measured or analyzed directly. Meteorites are extraterrestrial objects that can be retrieved from the surfaces of the Earth. Interplanetary dust particles can be collected from the upper atmosphere by high-flying aircrafts. Space probes have been sent to asteroids, comets and planetary satellites to measure their chemical compositions in situ. Samples have been retrieved from the Moon, asteroids, and comets and returned to earth for analysis.

Techniques used to analyze samples can be passive and non-destructive, e.g., through imaging and spectroscopy. Examples include nuclear magnetic resonance (NMR), fourier transform infrared spectroscopy (FTIR), X-ray absorption near edge spectroscopy (XANES), electron paramagnetic resonance (EPR), high resolution transmission electron microscopy (HRTEM). Alternatively, the samples can be altered by heating or dissolving in a solvent. The technique of pyrolysis gas chromatography heats a sample to release smaller molecules that can be separated and identified. The technique of secondary ion mass spectroscopy (SIMS) uses a beam of ions to sputter layers of sample and subject them to mass analysis.

\subsection{Meteorites}

When complex organics was first discovered in meteorites (Nagy et al. 1961), it took the scientific community by surprise. In the 50 years since, we have learned that almost all biologically relevant organic compounds have been found to be present in carbonaceous meteorites. In the soluble component of meteorites, over 14,000 compounds with millions of diverse structures have been identified. These include carboxylic acids, sulfonic and phosphonic acids, amino acids, aromatic hydrocarbons, heterocyclic compounds, aliphatic hydrocarbons, amines and amides, alcohols, aldehydes, ketones, and sugar related compounds (Schmitt-Kopplin et al. 2010). The decreasing abundance with increasing carbon number within the same class of compounds suggests that they are of abiotic origin.

Included in the soluble component of meteorites are over 100 amino acids (Pizzarello and Shock 2010), far more than the 20 present in proteins in biological organisms on Earth. Amino acids with both D (right-handed) and L (left-handed) chirality are represented in meteorites. This is clearly distinct from terrestrial amino acids where only L chirality is present in biological proteins (Pizzarello and Cronin 2000). The slight excess of L-amino acids observed in meteorites, if delivered to the Early Earth and amplified, may be responsible for the homochirality in terrestrial life 
(Aponte et al. 2014). The discovery of terrestrially rare nucleobases 2,6-diaminopurine and 6,8-diaminopurine in the meteorites suggests that there is a broader range of biochemistry possible in the Universe (Callahan et al. 2011).

Over $70 \%$ of the organics in carbonaceous chondrites are in the form of insoluble organic matter (IOM). The chemical structure of these insoluble macromolecular solids consists of small (1-4) aromatic rings, short aliphatic chains, and contain hetero elements such as O, S, and N (Derenne and Robert 2010; Cody et al. 2011). These structures are very similar to those of terrestrial kerogen and coal (Kerridge 1999; Sephton et al. 2004). In terms of abundance, the ratios of the atoms can be expressed as $\mathrm{C}_{100} \mathrm{H}_{46} \mathrm{~N}_{10} \mathrm{O}_{15} \mathrm{~S}_{4.5}$ (Pizzarello and Shock 2010).

The extensive varieties of organics in meteorites is evidence that abiotic synthesis of complex organics is possible in the extraterrestrial environment. What is not clear is whether these organics were made in the Solar System, or they were inherited from the interstellar medium. The excesses in $\mathrm{D}$ and ${ }^{13} \mathrm{C}$ abundance relative to the solar value are possible indicators of their interstellar origin.

\subsection{Comets}

Organics in comets are in the form of either gas-phase molecules (volatiles) or solid-state refractory organics. Gas-phase molecules can be detected either by $\mathrm{mm} /$ submm-wave or infrared spectroscopic observations. A large number of organic molecules consisting of $\mathrm{H}, \mathrm{C}, \mathrm{N}$, and $\mathrm{O}$ atoms have been detected. These include $\mathrm{CH}_{4}$, $\mathrm{C}_{2} \mathrm{H}_{2}, \mathrm{C}_{2} \mathrm{H}_{6}, \mathrm{CH}_{3} \mathrm{OH}, \mathrm{H}_{2} \mathrm{CO}, \mathrm{HOCH}_{2} \mathrm{CH}_{2} \mathrm{OH}, \mathrm{HCOOH}, \mathrm{HCOOCH} 3, \mathrm{CH}_{3} \mathrm{CHO}$, $\mathrm{NH}_{2} \mathrm{CHO}, \mathrm{NH}_{3}, \mathrm{HCN}, \mathrm{HNCO}, \mathrm{HNC}, \mathrm{CH}_{3} \mathrm{CN}, \mathrm{HC}_{3} \mathrm{~N}$ and others (Mumma and Charnley 2011). The Cometary Sampling and Composition (COSAC) mass spectrometer aboard Rosetta's Philae lander has detected an array of organic compounds, including methyl isocyanate $\left(\mathrm{CH}_{3} \mathrm{NCO}\right)$, acetone $\left(\mathrm{CH}_{3} \mathrm{COCH}_{3}\right)$, propanal $\left(\mathrm{C}_{2} \mathrm{H}_{5} \mathrm{CHO}\right)$, and acetamide $\left(\mathrm{CH}_{3} \mathrm{CONH}_{2}\right)$, on the surface of the comet 67P/Churyumov-Gerasimenko (Goesmann et al. 2015).

Since comets are sources of meteorites and interplanetary dust particles (IDPs), the organics similar to IOM are expected to be present in comets as well. Complex hydrocarbons are found in the samples returned by the Stardust mission to the Comet 81P/Wild 2 (Keller et al. 2006; Clemett et al. 2010). Figure 1 shows the $3.3 \mu \mathrm{m}$ aromatic and $3.4 \mu \mathrm{m} \mathrm{C}-\mathrm{H}$ stretching mode features observed in this comet are very similar to the unidentified infrared emission (UIE) bands found in the interstellar medium (Sect. 4.3). Some particles in the Stardust sample have been identified to be of interstellar origin (Westphal 2014). A broad 2.9-3.6 $\mu \mathrm{m}$ feature detected by the Visible InfraRed and Thermal Imaging Spectrometer (VIRTIS)on Rosetta is attributed to nonvolatile organic macromolecular materials on the nucleus of comet 67P/Churyumov-Gerasimenko (Capaccioni et al. 2015).

Among the biomolecules detected in the Stardust sample is glycine. Carbon isotopic ratios of the molecule suggests that it is of clear extraterrestrial origin (Elsila et al. 2009). 


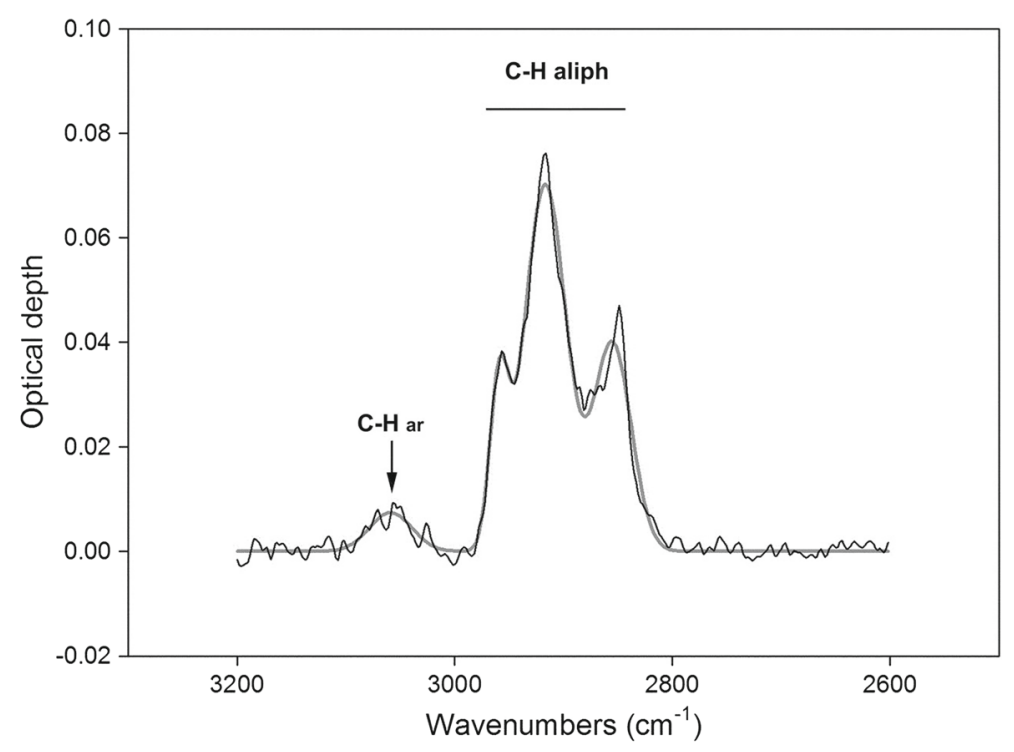

Fig. 1 Fourier transform infrared spectrum of materials from the Comet 81P/Wild 2 returned by the Stardust mission. Figure adapted from Keller et al. (2006)

\subsection{Asteroids}

Some asteroids have very red colors and low (0.01-0.15) albedos which are inconsistent with a mineral or ice composition. These properties are more compatible with complex organics, as macromolecular carbon compounds tend to have dark red, brown, or black colors (Gradie and Veverka 1980). Tholins, an artificial amorphous substance produced by UV photolysis of gas mixtures of $\mathrm{N}_{2}, \mathrm{NH}_{3}$, and $\mathrm{CH}_{4}$ (Sagan and Khare 1979), has been suggested as one of such possible organic constitutes of asteroids (Cruikshank and Imanaka 2005). The detections of the $3.4 \mu \mathrm{m}$ feature in the asteroids 24 Themis and 65 Cybele support the presence of complex organics on the surface of these asteroids (Campins et al. 2010; Licandro et al. 2011).

Samples returned from the near-Earth S-type asteroid Itokawa by the Hayabusa mission have been found to consist of mostly silicate minerals without any detectable amount of organics (Naraoka et al. 2015).

\subsection{Interplanetary dust particles}

Interplanetary dust particles (IDP) are micron-size fragments of comets and asteroids. IDPs are often carbon rich (up to $50 \%$ in weight), highly porous, and have not been significantly aqueously altered. As the Earth orbits the Sun, IDPs are swept up by the moving Earth and every year 20,000 to 60,000 tons of IDPs enter the Earth's atmosphere. These particles can be collected by high-flying aircraft and brought back to the laboratory for analysis. 
Fig. 2 The infrared absorption spectra of IDP L2008F7 and L2008G9 and the Murchison meteorite showing the aliphatic stretching modes of $\mathrm{CH}_{2}$ and $\mathrm{CH}_{3}$ (marked by vertical dashed lines). Figure adapted from Flynn et al. (2003)

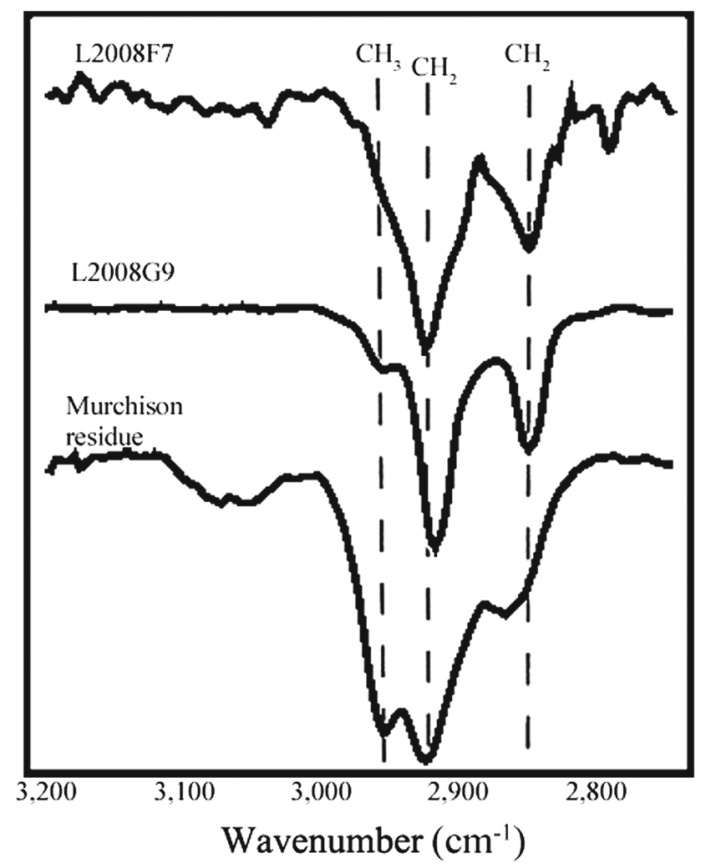

The chemical structures of IDPs can be determined by infrared spectroscopy, Raman spectroscopy, microprobe laser desorption mass spectrometry $\left(\mu \mathrm{L}^{2} \mathrm{MS}\right)$, and X-ray absorption near-edge structure spectroscopy (XANES). Complex aromatic compounds were first detected by $\mu \mathrm{L}^{2} \mathrm{MS}$ (Clemett et al. 1993). Infrared spectroscopy of IDPs shows aromatic and aliphatic 3.3 and $3.4 \mu \mathrm{m}$ features (Matrajt et al. 2013) (Fig. 2). Evidence of carbonate, oxygen, and nitrogen function groups can be found by XANES (Feser et al. 2003).

The large isotopic fractionation in the organic matter in IDPs $\left(\mathrm{D} / \mathrm{H}\right.$ and $\left.{ }^{15} \mathrm{~N} /{ }^{14} \mathrm{~N}\right)$ has been considered by some to represent preserved interstellar organic material (Messenger and Nakamura-Messenger 2015).

Micrometeorites are similar to IDP except in the collection method. Particles collected in icefields of Antarctica and Greenland are called micrometeorties, in contrast to IDPs which are collected in the upper atmosphere. For example, micrometeorties have been collected in Dome Fuji and Dome C in Antarctica by Japanese and French/Italian teams respectively. Analysis of the organic content of Antarctic micrometeorites shows excess amount of $\mathrm{N}$ over the typical $\mathrm{N}$ to $\mathrm{C}$ ratios observed in IOM. Since there is not yet any evidence for the existence of such N-rich solids in the interstellar medium, these organic compounds are likely to have formed in the outer regions of the early proto-planetary Solar System (Dartois et al. 2013).

\subsection{Planetary satellites}

Both the atmosphere and the surface of Titan are rich in complex organics. Molecular nitrogen and methane are the primary constituents of the Titan atmosphere. Other 
organic molecules observed include hydrocarbons, nitriles, and aromatics compounds. The Atacama Large Millimeter/submillimeter Array (ALMA), with its superior spatial resolution, is able to map the distribution of gas-phase molecules such as $\mathrm{C}_{2} \mathrm{H}_{5} \mathrm{CN}$, $\mathrm{HNC}, \mathrm{HC}_{3} \mathrm{~N}, \mathrm{CH}_{3} \mathrm{CN}$ and $\mathrm{CH}_{3} \mathrm{CCH}$ in the Titan atmosphere (Cordiner et al. 2015). The haze in the atmosphere of Titan is believed to be complex hydrocarbon-nitrile compounds. The most popular model of these compounds is tholins. The solar occultation spectra of the Titan atmosphere taken by the Cassini/VIMS instrument show distinct aliphatic signatures in the form of the $3.4 \mu \mathrm{m}$ feature (Kim et al. 2011). Similar features have also been observed in the atmosphere of Saturn (Kim et al. 2012). Numerous lakes of liquid methane and ethane, probably with other organics dissolved in them, are found on the surface of Titan. Hydrocarbon sand dunes have been estimated to cover $20 \%$ of Titan's surface (Lorenz et al. 2008).

\subsection{Minor planets and outer solar system objects}

It is now recognized that the outer Solar System contains a large population of icy objects, which have been referred to as either Kuiper Belt Objects or transneptunian objects (TNOs). The chemical constituents of TNOs are of particular interest because TNOs are believed to be the most primitive bodies in the Solar System. In addition to water ice, ices of $\mathrm{N}_{2}, \mathrm{CH}_{4}, \mathrm{CO}, \mathrm{CO}_{2}, \mathrm{C}_{2} \mathrm{H}_{6}$ and $\mathrm{HCN}$ also exist.

The most prominent member of the TNO family is Pluto. Recent images form the New Horizons spacecraft found that Pluto has a reddish brown color. Since ices are usually colorless, the color of Pluto may be due to complex organics on the surface (Materese et al. 2014). Between the orbits of Jupiter and Neptune minor planets with characteristics between asteroids and comets which are referred to as Centaurs. The colors and reflectance of Centaurs and TNOs have also been used to infer the presence of complex organics in TNOs (Giri et al. 2015).

\section{Organics as carriers of unidentified spectral phenomena in the ISM}

There are several outstanding mysteries in spectroscopic observations of the interstellar medium which could be traced to organic compounds as their carriers. The origin of diffuse interstellar bands (DIB), $217.5 \mathrm{~nm}$ feature, extended red emission (ERE), unidentified infrared emission (UIE) features, and the 21 and $30 \mu \mathrm{m}$ features have remained unsolved after decades of efforts. Because of the strengths and ubiquitous nature of the features, the carrier must be made up of common and abundant elements, with carbon being the most likely candidate.

It is interesting to note that these phenomena have been observed not only in the diffuse ISM, but also in circumstellar environments, in the galactic halo, and in external galaxies. In some cases they have been observed in galaxies with high redshifts, suggesting that the carriers responsible for these features were already present in the early Universe. 


\subsection{Diffuse interstellar bands}

The oldest of these mysteries are the diffuse interstellar bands (DIBs), which have been known for almost a century (Heger 1922). Although more than 500 bands from the near UV to near infrared have been detected, all except two have not been identified (Cami and Cox 2014). In the Milky Way Galaxy, DIBs have been seen towards over one hundred stars. In the Magellanic Clouds, DIBs have been seen in the spectrum of the supernova SN 1987A as well as in the spectra of reddened stars (Ehrenfreund et al. 2002). DIB carriers in the interstellar medium of external galaxies can be probed by supernovae (Sollerman et al. 2005), and DIBs have been detected in external galaxies with redshifts up to 0.5 (Sarre 2006).

DIBs are almost exclusively seen in absorption. The only exception is in the circumstellar envelope of HD 44179 (the Red Rectangle) where several DIB features are seen in emission (Winckel et al. 2002); see also Díaz-Luis et.al (2015) who reported evidence for circumstellar diffuse bands (both in emission and absorption) in the fullerene-containing PN Tc 1.

While the DIBs are widely accepted to be due to electronic transitions of gasphase C-based molecules, the exact chemical structures of the carrier(s) have not been identified. It has been said that DIBs represent the largest reservoir of organic material in the galaxy (Snow 2014). Polycyclic aromatic hydrocarbon (PAH) molecules have been widely discussed as possible candidates of carriers of DIBs (Léger and d'Hendecourt 1985; Salama and Ehrenfreund 2014). However, the electronic transitions of PAH molecules are well known but to date no visible-UV PAH transitions have been detected even after extensive searches (Gredel et al. 2011; Salama et al. 2011).

The most promising case is the identification of two DIBs at 963.2 and $957.7 \mathrm{~nm}$ with $\mathrm{C}_{60}^{+}$(Foing and Ehrenfreund 1994; Campbell et al. 2015). Two weaker lines of $\mathrm{C}_{60}^{+}$ at 942.8 and $936.6 \mathrm{~nm}$ were also identified with DIBs (Walker et al. 2015). Although the traditional view of the origin of the carriers of DIBs is that they are synthesized and the study of DIBs in C60-rich PNe (García-Hernández and Díaz-Luis 2013) from small gas-phase molecule precursors, the identification of two DIBs with $\mathrm{C}_{60}^{+}$suggests the possibility that the DIB carrier molecules may represent breakdown products of large, complex organic compounds, such as mixed aromatic/aliphatic organic nanoparticles (MAON) (Kwok 2014) or other amorphous hydrocarbons (Sect. 7).

\subsection{The $220 \mathrm{~nm}$ feature}

The $220 \mathrm{~nm}$ extinction feature has been known for about 50 years (Stecher 1965). It was extensively observed by the International Ultraviolet Explorer (IUE) satellite and is found to have a remarkable constancy in its peak wavelength of $217.5 \mathrm{~nm}$, corresponding to an energy gap of $5.7 \mathrm{eV}$. This is not just a local phenomenon as the feature has been detected in galaxies as distant as redshift $>2$ (Elíasdóttir et al. 2009).

The carrier of the $220 \mathrm{~nm}$ feature is often attributed to a carbonaceous solid such as amorphous carbon (Mennella et al. 1998), carbon onions (Iglesias-Groth 2004), hydrogenated fullerences (Cataldo and Iglesias-Groth 2009), or polycrystalline graphite (Papoular and Papoular 2009). The detection of the $220 \mathrm{~nm}$ feature in IDPs with 
monocrhomated electron energy-loss spectroscopy suggests a possible interstellarsolar system connection (Bradley et al. 2005).

\subsection{Unidentified infrared emission bands}

A family of unidentified infrared emission (UIE) features at 3.3, 6.2, 7.7, 8.6, and $11.3 \mu \mathrm{m}$ was discovered almost 40 years ago (Russell et al. 1977). The $3.3 \mu \mathrm{m}$ feature was first identified as the $\mathrm{C}-\mathrm{H}$ stretching mode of aromatic compounds by Knacke (1977). The organic affiliation was extensively discussed by Duley and Williams (1981), who assigned the 3.3 and $11.3 \mu \mathrm{m}$ features to graphitic (aromatic) materials. Specifically, the $3.3 \mu \mathrm{m}$ feature is identified as due to aromatic $\mathrm{C}-\mathrm{H}$ stretch, the $6.2 \mu \mathrm{m}$ feature as aromatic $\mathrm{C}-\mathrm{C}$ stretch, the $8.6 \mu \mathrm{m}$ feature as the $\mathrm{C}-\mathrm{H}$ in-plane bending mode, and the $11.3,12.4$ and $13.3 \mu \mathrm{m}$ features as due to solo, duo and trio $\mathrm{C}-\mathrm{H}$ out-of-plane bending modes, respectively.

Also present in astronomical spectra are emission features around $3.4 \mu \mathrm{m}$, which arise from symmetric and anti-symmetric $\mathrm{C}-\mathrm{H}$ stretching modes of methyl and methylene groups (Puetter et al. 1979; Geballe et al. 1992). The bending modes of these groups also manifest themselves at 6.9 and $7.3 \mu \mathrm{m}$ (Jourdain de Muizon et al. 1990; Chiar et al. 2000). In addition, there are unidentified emission features at 15.8, $16.4,17.4,17.8$, and $18.9 \mu \mathrm{m}$, which have been observed in proto-planetary nebulae, reflection nebulae, and galaxies.

The emission bands themselves are often accompanied by strong, broad emission plateaus features at $6-9,10-15$, and $15-20 \mu \mathrm{m}$. The first two plateau features have been identified as superpositions of in-plane and out-of-plane bending modes emitted by a mixture of aliphatic side groups attached to aromatic rings (Kwok et al. 2001). The 15-20 $\mu \mathrm{m}$ plateau feature has been detected in young stellar objects, compact HII regions, and planetary nebulae, but are especially strong in some proto-planetary nebulae (Zhang et al. 2010). A possible origin of this broad feature arises from CCC in- and out-of-plane bending of aromatic rings (Kerckhoven et al. 2000).

The UIE features are always associated with a strong emission continuum which cannot be explained by free-free emission or reflected starlight. For example, scattered starlight is expected to be strongly polarized, but the observed continuum emission is unpolarized. In the diffuse interstellar medium, the strength of the UIE features is strongly correlated with the dust continuum, suggesting that the emission bands are physically related to the continuum (Kahanpää et al. 2003).

The UIE features are seen in very different radiation environments and their emission peak wavelengths and profiles also vary. The energy sources responsible for the excitation of the features have temperatures ranging from tens of thousands of degrees in planetary nebulae (the central star temperature of NGC 7027 is $200,000 \mathrm{~K}$ ), to $\sim 30,000 \mathrm{~K}$ in HII regions, and to only thousands of degrees in reflection nebulae and proto-planetary nebulae. The UIE features seen in the reflection nebula NGC 7023 are very similar to those seen in the planetary nebula NGC 7027 in spite of the very different intensities of UV background in the two nebulae.

A variety of chemical structures have been suggested as the carriers of the UIE bands. These include PAH molecules (Léger and Puget 1984; Allamandola et al. 1989), 


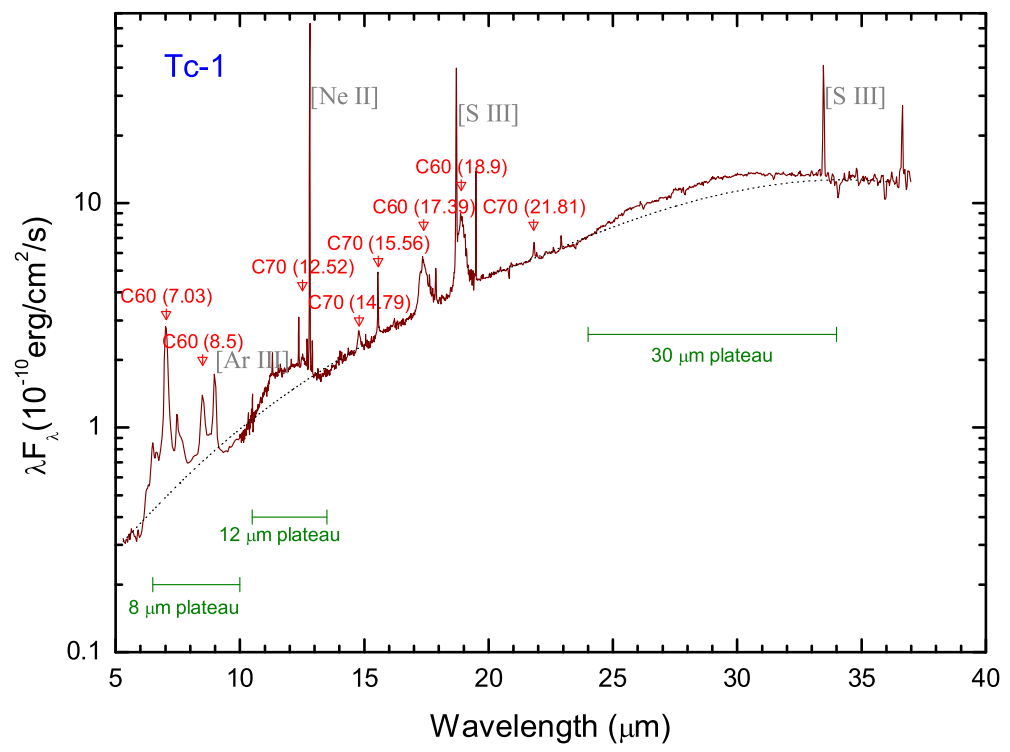

Fig. 3 The Spitzer IRS spectrum of planetary nebula Tc-1 shows $\mathrm{C}_{60}$ and $\mathrm{C}_{70}$ emission bands (in red) as well as broad emission plateau features at 8,12 , and $30 \mu \mathrm{m}$ (in green). The narrow lines are atomic lines (in grey). No UIE bands are seen in this object

small carbonaceous molecules (Bernstein and Lynch 2009), hydrogenated amorphous carbon (HAC), soot and carbon nanoparticles (Hu and Duley 2008), quenched carbonaceous composite particles (QCC, Sakata et al. 1987), kerogen and coal (Papoular et al. 1989), petroleum fractions (Cataldo et al. 2002), and mixed aromatic/aliphatic organic nanoparticles (MAON, Kwok and Zhang 2011, 2013). Among these proposed carriers, the PAH hypothesis is by far the most popular. However, the PAH hypothesis has a number of difficulties and its validity has been put into question (Zhang and Kwok 2015; Sadjadi et al. 2015).

The discovery of fullerene $\left(\mathrm{C}_{60}\right)$ in the planetary nebula Tc-1 (Cami et al. 2010) has generated interests in possible links between $\mathrm{C}_{60}$ and the UIE phenomenon. Although Tc-1 has no UIE features, it does show the 8 and $12 \mu \mathrm{m}$ plateaus (Fig. 3). The protoplanetary nebula IRAS $01005+7910$, however, show $\mathrm{C}_{60}$ features, UIE bands, as well as the 8, 12, and $17 \mu \mathrm{m}$ emission plateau features (Zhang and Kwok 2011). The association between $\mathrm{C}_{60}$ and the 8 and $12 \mu \mathrm{m}$ plateau features (Zhang and Kwok 2013; Otsuka et al. 2013) suggests that amorphous carbonaceous solids such as MAONs could be precursors of fullerenes (García-Hernández et al. 2012; Bernard-Salas et al. 2012). PAHs have also been proposed as the $\mathrm{C}_{60}$ precursors (Berné and Tielens 2012). But the dehydrogenation of PAHs induced by UV photons is more difficult than that of MAONs because the photon energy can quickly spread out within the aromatic nets.

\subsection{Extended red emission}

The observation of the Extended Red Emission (ERE) also goes back 30 years. ERE is commonly seen in reflection nebulae (Witt and Schild 1988; Witt and Boroson 
1990), but has also been detected in dark nebulae, cirrus clouds, planetary nebulae, HII regions, the diffuse interstellar medium, and in haloes of galaxies. The central wavelength of the emission shifts from object to object, or even between locations within the same object. Other than the fact that it may be due to photoluminescence, exact nature of its carrier is still unknown. Other unidentified optical emissions include a set of bright visible bands seen alongside ERE in the Red Rectangle nebula (Schmidt and Witt 1991) and blue luminescence which is seen both in this object and a number of other sources (Vijh et al. 2004).

If the ERE is a result of photoluminescience, the carrier is likely to be a semiconductor with a nonzero band gap and silicon nanoparticles have been suggested as a candidate (Ledoux et al. 1998; Witt et al. 1998). Other possibilities include QCC (Sakata et al. 1992), $\mathrm{C}_{60}$ (Webster 1993), and nanodiamonds (Chang et al. 2006).

\subsection{The 21 and $30 \mu \mathrm{m}$ features}

The 21 and $30 \mu \mathrm{m}$ unidentified infrared features (Fig. 4) are generally associated with objects in the late stages of stellar evolution (Kwok et al. 1989; Forrest et al. 1981), and can be responsible for a significant fraction (up to $8 \%$ for the $21 \mu \mathrm{m}$ feature and $20 \%$ for the $30 \mu \mathrm{m}$ feature) of the energy output of the stellar sources (Hrivnak et al. 2000). High resolution $I S O$ observations have found that the $21 \mu \mathrm{m}$ features have the same intrinsic profile and peak wavelength $(20.1 \mu \mathrm{m})$ (Volk et al. 1999). There is no evidence for any discrete sub-structure due to molecular bands in the observed spectra, suggesting that the $21-\mu \mathrm{m}$ feature is either due to a solid substance or a mixture

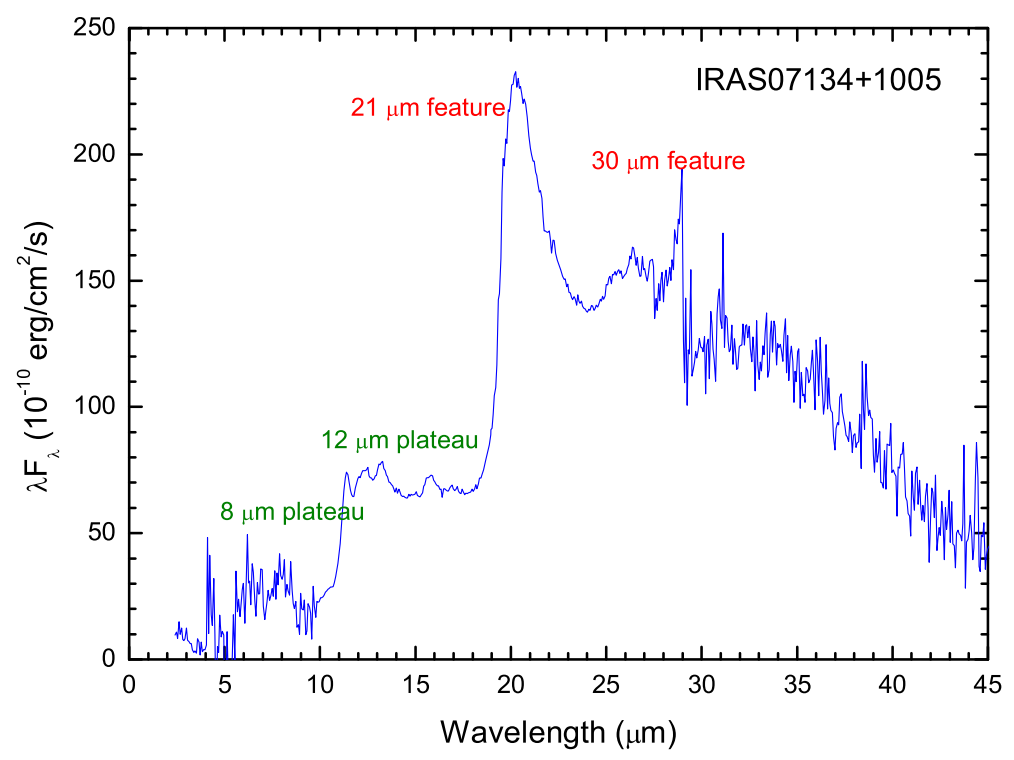

Fig. 4 Combined ISO SWS and LWS and Spitzer IRS spectra of the proto-planetary nebula IRAS 07134+1005 showing the 21 and 30 unidentified emission features. Also seen in the spectra are broad plateau emission features around 8 and $12 \mu \mathrm{m}$. All these features sit on top of a strong continuum 
of many similarly structured large molecules. These features have been detected in evolved stars in the Magellanic Clouds (Volk et al. 2011).

Many proposals for the origin of the $21 \mu \mathrm{m}$ feature have been made, including hydrogenated fullerenes (Webster 1995), SiC (Speck and Hofmeister 2004), and thiourea groups attached to aromatic/aliphatic structures (Papoular 2011). The first suggestion of the origin of the $30 \mu \mathrm{m}$ feature is $\mathrm{MgS}$, but it is now considered as unlikely based on abundance grounds (Zhang et al. 2009). Since the $30 \mu \mathrm{m}$ feature is exclusively seen in carbon-rich objects, an organic carrier is much more plausible. The common correlation between the 21 and $30 \mu \mathrm{m}$ features with the UIE bands suggests that these phenomena may be related.

\section{Organics in the diffuse ISM}

The detection of the $3.4 \mu \mathrm{m}$ in absorption has led to the realization of the existence of an aliphatic component in the diffuse interstellar medium (Wickramasinghe and Allen 1980). This feature is a superposition of symmetric and anti-symmetric C-H stretching modes arising from methyl $\left(-\mathrm{CH}_{3}\right)$ and methylene $\left(-\mathrm{CH}_{2}\right)$ groups in aliphatic hydrocarbons. Since the initial discovery, the $3.4 \mu \mathrm{m}$ feature has been observed along the line of sight to a number of infrared background sources (Pendleton et al. 1994). High spectral resolution observations are able to resolve the feature into individual $\mathrm{CH}_{2}$ and $\mathrm{CH}_{3}$ symmetric and antisymmetric stretching modes (Dartois et al. 2007) (Fig. 5).

Since the derivation of column densities from the strengths of absorption features does not depend on an excitation model, the overall abundance of aliphatic compounds

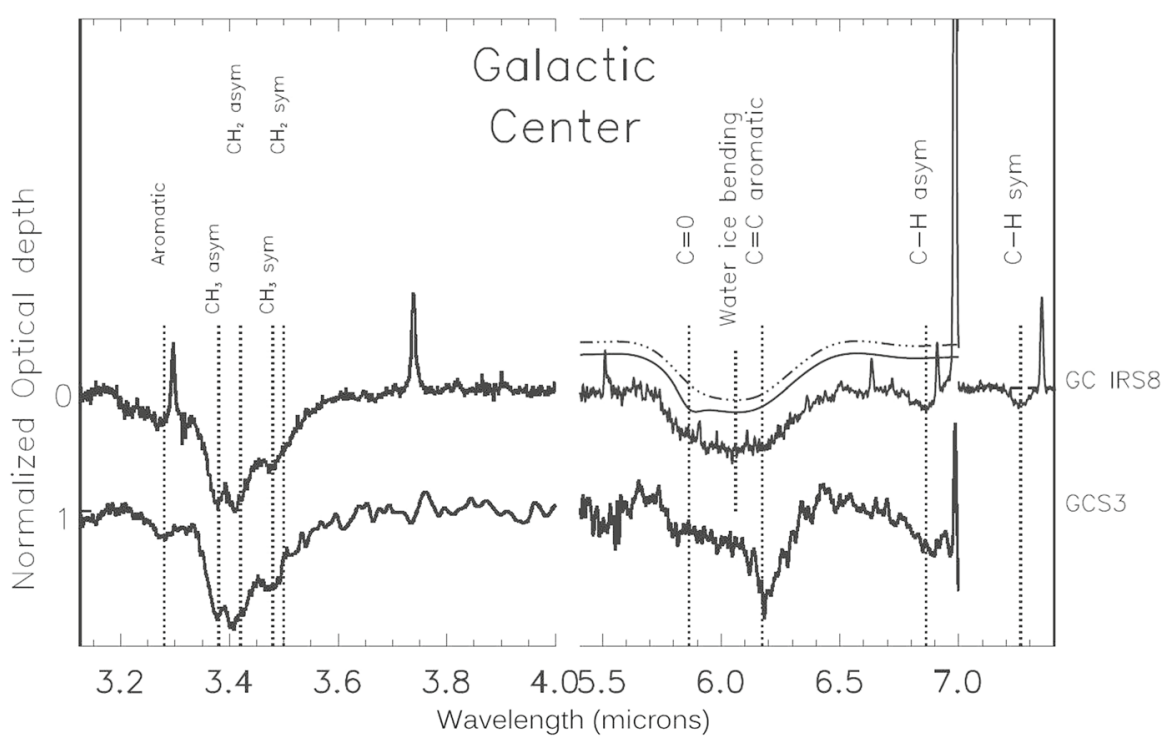

Fig. 5 The $3.4 \mu \mathrm{m}$ feature is seen in absorption against the background infrared continuum along two lines of sight (GC IRS8 and GCS3) towards the galactic center. Figure adapted from Dartois (2011) 
can be quite reliably determined. The strength of the $3.4 \mu \mathrm{m}$ feature suggests that at least $15 \%$ of all the carbon atoms are in the form of aliphatic compounds (Dartois 2011).

\section{Organics in external galaxies}

The UIE bands have been detected in planetary nebulae, reflection nebulae, HII regions, diffuse interstellar medium, and external galaxies. In some active galaxies, up to $20 \%$ of the total luminosity of the galaxy is emitted in the UIE bands (Smith et al. 2007) (Fig. 6). The detection of UIE bands in high-redshift galaxies (Teplitz et al. 2007) and quasars (Lutz et al. 2007) implies that complex organics were widely present as early as 10 billion years ago. This suggests that abiological synthesis of complex organics has been occurring through most of the history of the Universe.

A survey of 150 luminous and ultraluminous galaxies by the AKARI satellite has resulted in common detection of the $3.3 \mu \mathrm{m}$ emission feature. The observed fraction of total energy emitted in the $3.3 \mu \mathrm{m}$ band is $\sim 10^{-3}$ (Imanishi et al. 2010).

In addition, the 3.4 and $6.9 \mu \mathrm{m}$ aliphatic $\mathrm{C}-\mathrm{H}$ stretching and bending modes have been detected in absorption in our own and other galaxies. Strong $3.4 \mu \mathrm{m}$ absorption features can be seen in absorption in ultraluminous infrared galaxies (Mason et al. 2004; Imanishi et al. 2010). Figure 7 shows the $3.4 \mu \mathrm{m}$ feature in absorption next to the $3.3 \mu \mathrm{m}$ emission feature (Risaliti et al. 2006).

Since the UIE bands are commonly used to trace star formation and to probe the radiation field of galaxies assuming that $\mathrm{PAH}$ molecules are the carrier (Valiante et al.

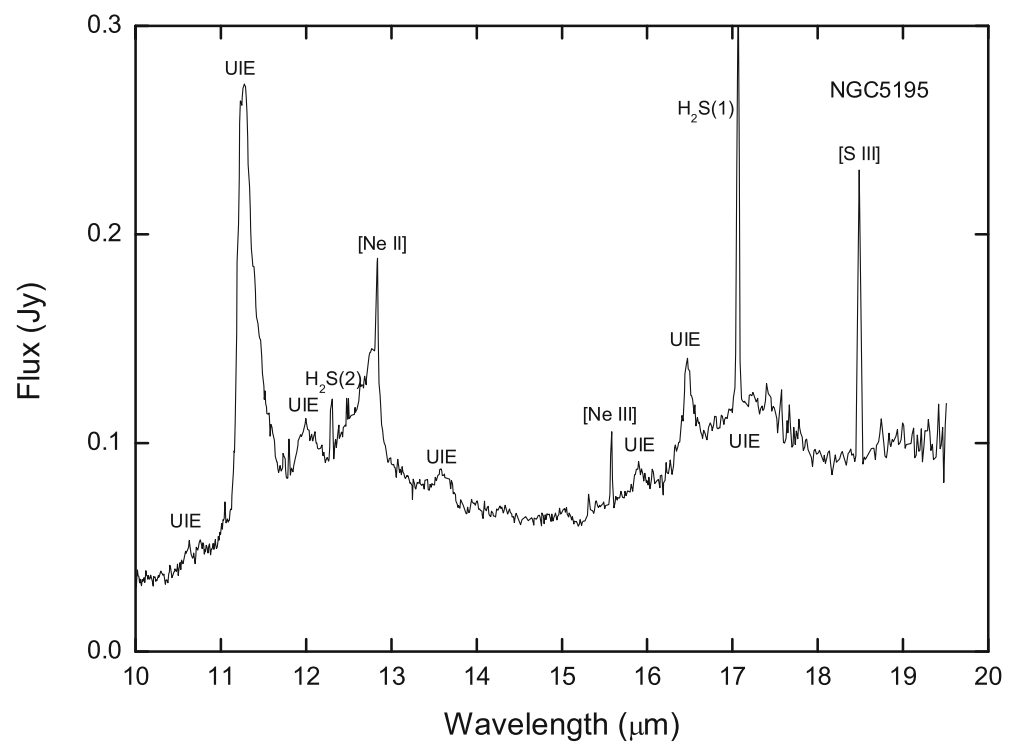

Fig. 6 The Spitzer IRS spectrum of the galaxy NGC 5195 showing strong UIE features at 11.3, 16.4 $\mu \mathrm{m}$ in addition to the atomic and molecular hydrogen lines 


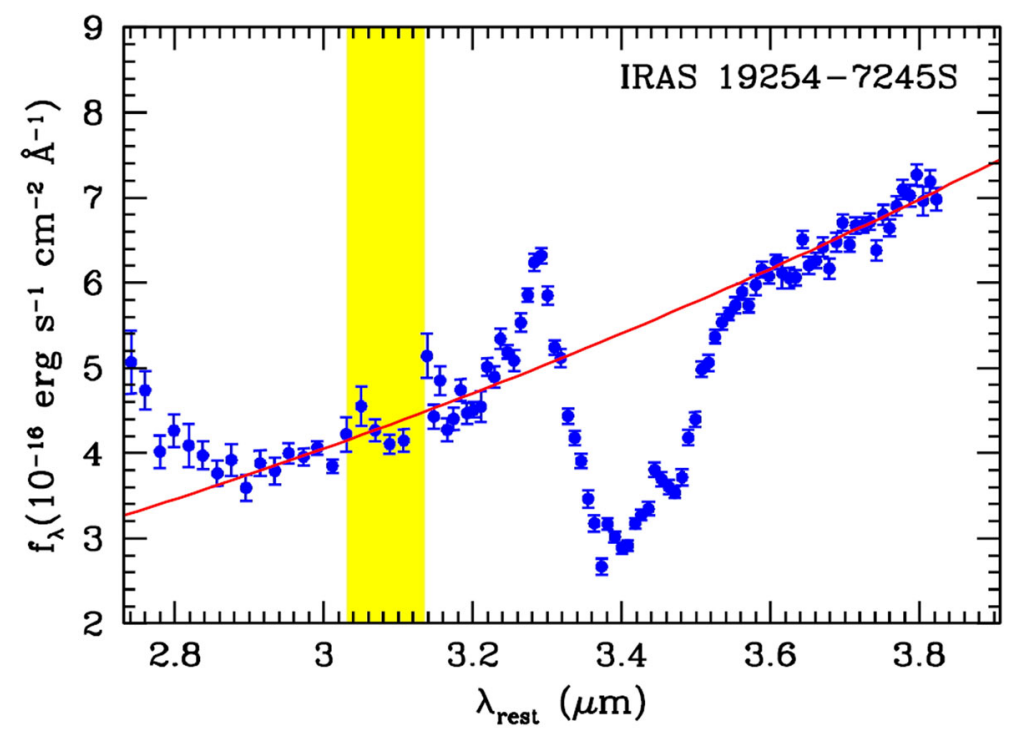

Fig. 7 Infrared spectrum of ultraluminous infrared galaxy IRAS 19254-7245S obtained with the VLT Infrared Spectrometer and Array Camera. The yellow band indicates spectral region of bad atmospheric transmission. Figure from Risaliti et al. (2006)

2007; Pope et al. 2013), a correct identification of the UIE bands are essential for our understanding of the galactic environment.

\section{Laboratory simulations of interstellar organics}

In order to identify possible carriers of the unidentified spectral phenomena discussed in Sect. 4, it would be useful to see what carbonaceous products can naturally exist in the interstellar medium. By introducing $\mathrm{H}$ into graphite $\left(s p^{2}\right)$ and diamond $\left(s p^{3}\right)$, a variety of amorphous C-H alloys can be created (Cataldo 2004; Jones 2012a, b, c; Jones et al. 2013). Different geometric structures with long- and short-range can be created by varying the aromatic to aliphatic ratios. A schematic of possible structures of amorphous carbonaceous solids is shown in Fig. 8. The lower left corner of the triangle represents graphite, the top corner represents diamonds, PAHs are on the bottom edge, and various forms of amorphous hydrogenated carbon can exist in the interior of the triangle. The infrared spectra of these amorphous carbonaceous materials (Dischler et al. 1983) resemble the astronomical UIE bands seen in planetary nebulae and proto-planetary nebulae (Fig. 9). Since these amorphous carbonaceous solids have absorption bands in the visible, they can be easily excited by visible light from stars.

Organic particles of such structures are natural products of combustion. The first nucleation products for soot particles formed in flames have structures consisting of islands of aromatic rings linked by chains. Identified sidegroups in soot include methyl $\left(-\mathrm{CH}_{3}\right)$, methylene $\left(-\mathrm{CH}_{2}\right)$, carbonyl $(\mathrm{C}=\mathrm{O})$, aldehydic $(-\mathrm{HCO})$, phenolic $(-\mathrm{OH})$, 


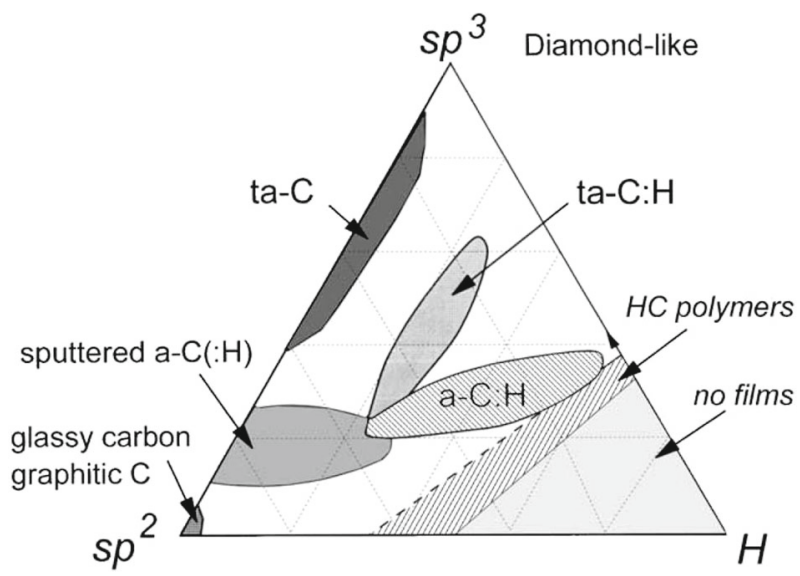

Fig. 8 Phase diagram of amorphous carbon based on pure $\mathrm{C}$ and $\mathrm{H}$ compounds. The lower right corner of the triangle represents pure $\mathrm{H}$, lower left corner pure graphite-like $\left(s p^{2}\right)$ materials (C rings on a plane with no $\mathrm{H}$ ), and the upper corner pure diamond ( $s p^{3}, \mathrm{C}$ arranged in tetrahedral forms). Areas inside the triangle represent various $\mathrm{H} / \mathrm{C}$ ratios and $s p^{2} / s p^{3}$ mixed hybridization states. Figure adapted from Robertson (2002)
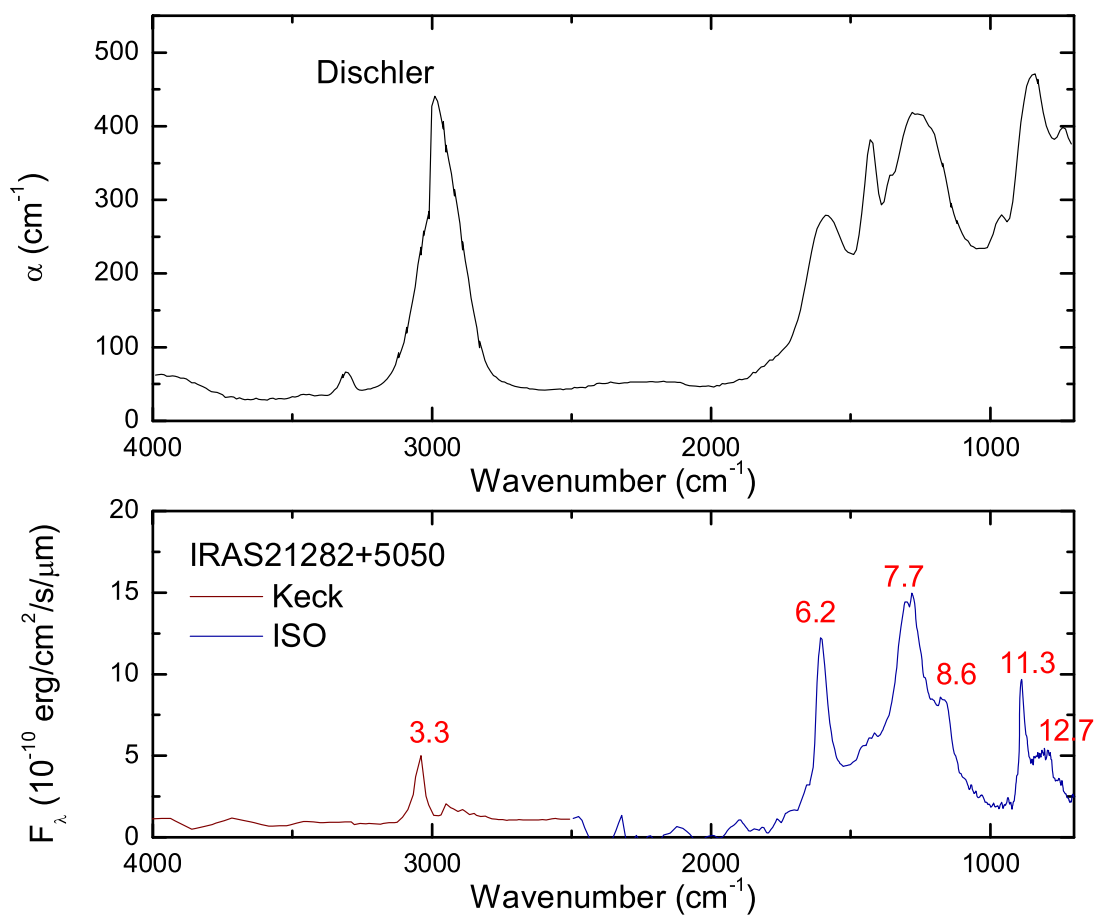

Fig. 9 Laboratory infrared spectra of hydrogenated amorphous carbon (top Dischler et al. (1983) compared to the astronomical spectrum of the planetary nebula IRAS 21282+5050 (bottom panel). The UIE bands are labeled by their wavelengths in $\mu \mathrm{m}$ 
and amino (- $\left.\mathrm{NH}_{2}\right)$ groups (Pino et al. 2008). Similar combustion processes may be at work in carbon-rich circumstellar envelopes.

By the early 1980s, it was known that carbon clusters can be produced by laser vaporization of graphite followed by supersonic expansion into an inert gas. The employment of this technique has led to the discovery of fullerene, a new form of carbon (Kroto et al. 1985). Since then, various techniques based on laser pyrolysis of gas-phase hydrocarbons followed by condensation have been used to create laboratory counterparts of cosmic organic dust (Jäger et al. 2009). These include the quenching of plasma of 4-torr methane (Sakata et al. 1987), hydrocarbon flame or arc-discharge in a neutral of hydrogenated atmosphere (Colangeli et al. 2003; Mennella et al. 2003), laser ablation of graphite in a hydrogen atmosphere (Scott and Duley 1996; Mennella et al. 1999), infrared laser pyrolysis of gas phase molecules $\left(\mathrm{C}_{2} \mathrm{H}_{4}, \mathrm{C}_{4} \mathrm{H}_{6}\right)($ Herlin et al. 1998), photolysis of methane at low temperatures (Dartois et al. 2004), and flame combustion of $\mathrm{C}_{2} \mathrm{H}_{2}, \mathrm{C}_{2} \mathrm{H}_{4}, \mathrm{C}_{3} \mathrm{H}_{6}$ mixed with $\mathrm{O}_{2}$, forming soot (Pino et al. 2008).

In addition to soot, there are also other natural products such as coal and kerogen that have similar amorphous mixed aromatic/aliphatic properties (Guillois et al. 1996; Papoular 2001). The infrared spectra of petroleum and asphaltenes also show spectral features similar to astronomical UIE bands (Cataldo et al. 2002, 2013).

\section{Origin and distribution of complex organics in the Universe}

Complex organics are now known to be widely present in the Universe, from Solar System objects to distant galaxies. These organics are synthesized abiologically, and do not represent breakdown products of living organisms. In contrast, almost all of the complex organics on Earth are biological in origin, making the Earth an exception to the wide presence of abiological organics in the Universe.

Although the exact pathways of synthesis are not known, it is widely believed that these complex organics are synthesized from simple organic molecules, either through gas-phase reactions or chemical reactions on solid-state surfaces. In the astronomical community, there is a lot of interest on the possibility of organic synthesis taking place in star forming regions, as there are heavy concentrations of gas-phase organics in these objects. In the space science community, the origin of complex organics in Solar System objects is often traced to the early epochs of the Solar System, although transport from interstellar medium to the primordial solar nebula has also been discussed.

The only concrete observational evidence for organic synthesis exists in circumstellar envelopes. During the asymptotic-giant-branch phase of evolution, the element carbon is produced by triple- $\alpha$ reactions in the stellar core and brought to the surface via convection. Due to the extended atmosphere and low surface temperatures of these stars, simple molecules such as $\mathrm{C}_{2}, \mathrm{C}_{3}$, and $\mathrm{CN}$ are formed in the stellar atmosphere and ejected by a stellar wind. In the circumstellar envelopes formed by stellar wind outflows, over 80 gas-phase molecules, including organics $\left(\mathrm{C}_{2} \mathrm{H}_{2}\right.$, $\left.\mathrm{CH}_{4}, \mathrm{H}_{2} \mathrm{CO}, \mathrm{CH}_{3} \mathrm{CN}\right)$, radicals $\left(\mathrm{C}_{2} \mathrm{H}, \mathrm{C}_{3}, \mathrm{HCO}^{+}\right)$, rings $\left(\mathrm{C}_{3} \mathrm{H}_{2}\right)$, and chains $\left(\mathrm{HC}_{3} \mathrm{~N}\right.$, $\mathrm{HC}_{5} \mathrm{~N}, \mathrm{HC}_{7} \mathrm{~N}$ ), are synthesized. All these molecules are formed within the dynamical timescales of the outflow, which is $\sim 10^{4}$ years. After the stellar wind completely 
deplete the stellar envelope, and circumstellar environment is exposed to increasingly more energetic radiation field as the stellar core is gradually exposed. It is at this stage that aromatic and aliphatic compounds, as well as fullerenes are formed. We therefore have extremely strong observational constraints that complex organics are formed in a low-density environment over very short $\left(10^{3}\right.$ years) time scales (Kwok 2004).

Since over $90 \%$ of all stars in the Galaxy go through this stage of evolution, a very large amount of complex organics are created by stars. We also know that these organic products are distributed to the interstellar medium through stellar winds. Whether these organic materials are destroyed or further processed in the interstellar medium, we do not know. It would not be unreasonable to assume that the early Solar System inherited some of these stellar material, as the IOM of meteorites show remarkable similarities to stellar produced organics.

Since the organic signatures are particularly strong in ultraluminous galaxies, it is natural to assume that the synthesis and/or excitation of organics in galaxies are related to the star formation process. Since the spectra of galaxies represent integrated light output from the entire galaxy, we do not have any information on the spatial distribution or origin of such organics. We do know, however, that organic synthesis happened in the Universe almost as soon as carbon is synthesized, and organic synthesis is common in the Universe.

The inner Solar System, including the early Earth, was extensively bombarded by external objects between 4.5 to 3.8 billion years ago. This has led to speculations on externally delivered organics to Earth may have influenced the origin of life on Earth (Anders 1989; Chyba and Sagan 1992). It is estimated that the amount of extraterrestrial organics delivered to Earth by comets, asteroids, and IDPs during the heavy bombardment period exceed those created from terrestrial sources through UV photolysis, electric discharge or from hydrothermal vents by several orders of magnitude (Ehrenfreund et al. 2002). To what extent that these extraterrestrial organics can survive intact during delivery is dependent on the mechanism of energy loss during the passage through the atmosphere. Energy loss through radiation or ablation has to exceed that of thermal decomposition of organics in order for the organics to remain intact upon impact. It is expected that smaller particles such as IDP may be more efficient in the delivery of organics (Pasek 2016).

If external delivery of organics played a major role in the development of life on Earth, then similar circumstances will also apply to other planetary systems in the Galaxy. Since planetary systems are now known to be common in our Galaxy (Winn and Fabrycky 2015), similar organic enrichment by stellar ejecta may have occurred in other planetary systems.

\section{Outlook}

Although the wide presence of extraterrestrial complex organics was not expected just a couple decades ago, observational evidence has completely changed our preconceived theoretical notions. With new large millimeter-wave arrays such as ALMA, more and more complex gas-phase organics will likely be detected, including possibly simple amino acids and building blocks of carbohydrates, lipids and nucleic acids. For a 
better determination of the chemical structure of organics in stars, interstellar clouds and galaxies, we need infrared space missions capable of high spectroscopic resolution observations. Space missions to Mars, planetary satellites, comets and asteroids have the potential of direct detection of complex organics in an ever widening types of environments. All these observations and measurements need to be accompanied by extensive laboratory analysis and simulations for correct identification of the chemical species. The study of complex organics in space has significant implications on the origin of life on Earth as well as our perception of our place in the Universe.

Acknowledgements I would like to thank my collaborators Yong Zhang and SeyedAbdolreza Sadjadi for their contributions. I also thank Dale Cruikshank, Bill Irvine, and Masatoshi Ohishi for their helpful comments on earlier drafts of the manuscript, The Laboratory for Space Research was established by a special Grant from the University Development Fund of the University of Hong Kong. This work is also in part supported by a Grant from the HKRGC (HKU 7027/11P).

Open Access This article is distributed under the terms of the Creative Commons Attribution 4.0 International License (http://creativecommons.org/licenses/by/4.0/), which permits unrestricted use, distribution, and reproduction in any medium, provided you give appropriate credit to the original author(s) and the source, provide a link to the Creative Commons license, and indicate if changes were made.

\section{References}

Allamandola LJ, Tielens AGGM, Barker JR (1989) Interstellar polycyclic aromatic hydrocarbons: the infrared emission bands, the excitation/emission mechanism and the astrophysical implications. Astrophys J Suppl Ser 71:733-775

Anders E (1989) Pre-biotic organic matter from comets and asteroids. Nature 342:255-257

Aponte JC, Dworkin JP, Elsila JE (2014) Assessing the origins of aliphatic amines in the Murchison meteorite from their compound-specific carbon isotopic ratios and enantiomeric composition. Geochimica et Cosmochimica Acta 141:331-345

Belloche A, Menten KM, Comito C, Müller HSP, Schilke P, Ott J, Thorwirth S, Hieret C (2008) Detection of amino acetonitrile in Sgr B2(N). Astron Astrophys 482:179-196

Bernard-Salas J, Cami J, Peeters E, Jones AP, Micelotta ER, Groenewegen MAT (2012) On the excitation and formation of circumstellar fullerenes. Astrophys J 757:41

Berné O, Tielens AGGM (2012) Formation of buckminsterfullerene (C60) in interstellar space. Proc Natl Acad Sci 109:401-406

Bernstein LS, Lynch DK (2009) Small carbonaceous molecules, ethylene oxide $\left(\mathrm{c}-\mathrm{C}_{2} \mathrm{H}_{4} \mathrm{O}\right)$ and cyclopropenylidene $\left(\mathrm{c}-\mathrm{C}_{3} \mathrm{H}_{2}\right)$ : sources of the unidentified infrared bands? Astrophys J 704(1):226-239

Bradley J, Dai ZR, Erni R, Browning N, Graham G, Weber P, Smith J, Hutcheon I, Ishii H, Bajt S, Floss C, Stadermann F, Sandford S (2005) An astronomical $2175 \AA$ feature in interplanetary dust particles. Science 307(5707):244-247

Callahan MP, Smith KE, Cleaves HJ, Ruzicka J, Stern JC, Glavin DP, House CH, Dworkin JP (2011) Carbonaceous meteorites contain a wide range of extraterrestrial nucleobases. Proc Natl Acad Sci 108(34):13995-13998

Cami J, Cox NLJ (2014) IAU symposium 297: the diffuse interstellar bands, CUP

Cami J, Bernard-Salas J, Peeters E, Malek SE (2010) Detection of $\mathrm{C}_{60}$ and $\mathrm{C}_{70}$ in a young planetary nebula. Science 329:1180-1182

Campbell EK, Holz M, Gerlich D, Maier JP (2015) Laboratory confirmation of C60+ as the carrier of two diffuse interstellar bands. Nature 523(7560):322-323

Campins H, Hargrove K, Pinilla-Alonso N, Howell ES, Kelley MS, Licandro J, Mothé-Diniz T, Fernández Y, Ziffer J (2010) Water ice and organics on the surface of the asteroid 24 Themis. Nature 464(7293):13201321

Capaccioni F, Coradini A, Filacchione G, Erard S, Arnold G, Drossart P, De Sanctis MC, Bockelee-Morvan D, Capria MT, Tosi F, Leyrat C, Schmitt B, Quirico E, Cerroni P, Mennella V, Raponi A, Ciarniello M, McCord T, Moroz L, Palomba E, Ammannito E, Barucci MA, Bellucci G, Benkhoff J, Bibring JP, 
Blanco A, Blecka M, Carlson R, Carsenty U, Colangeli L, Combes M, Combi M, Crovisier J, Encrenaz T, Federico C, Fink U, Fonti S, Ip WH, Irwin P, Jaumann R, Kuehrt E, Langevin Y, Magni G, Mottola S, Orofino V, Palumbo P, Piccioni G, Schade U, Taylor F, Tiphene D, Tozzi GP, Beck P, Biver N, Bonal L, Combe J-P, Despan D, Flamini E, Fornasier S, Frigeri A, Grassi D, Gudipati M, Longobardo A, Markus K, Merlin F, Orosei R, Rinaldi G, Stephan K, Cartacci M, Cicchetti A, Giuppi S, Hello Y, Henry F, Jacquinod S, Noschese R, Peter G, Politi R, Reess JM, Semery A (2015) The organic-rich surface of comet 67P/Churyumov-Gerasimenko as seen by VIRTIS/Rosetta. Science 347:0628

Carroll PB, Drouin BJ, Widicus Weaver SL (2010) The submillimeter spectrum of glycolaldehyde. Astrophys J 723:845-849

Caselli P, Ceccarelli C (2012) Our astrochemical heritage. A A Rev 20:56

Cataldo F (2004) From elemental carbon to complex macromolecular networks in space. In: Ehrenfreund P (ed) Astrobiology: future perspective. Kluwer, Dordrecht, pp 97-126

Cataldo F, Iglesias-Groth S (2009) On the action of UV photons on hydrogenated fulleranes $\mathrm{C}_{60} \mathrm{H}_{36}$ and $\mathrm{C}_{60} \mathrm{D}_{36}$. Mon Not R Astron Soc 400:291-298

Cataldo F, Keheyan Y, Heymann D (2002) A new model for the interpretation of the unidentified infrared bands (UIBS) of the diffuse interstellar medium and of the protoplanetary nebulae. Int $\mathbf{J}$ Astrobiol $1: 79-86$

Cataldo F, García-Hernández DA, Manchado A (2013) Far- and mid-infrared spectroscopy of complex organic matter of astrochemical interest: coal, heavy petroleum fractions and asphaltenes. Mon Not R Astron Soc 429:3025-3039

Chang H-C, Chen K, Kwok S (2006) Nanodiamond as a possible carrier of extended red emission. Astrophys J 639:L63-L66

Charnley SB, Kuan Y-J, Huang H-C, Botta O, Butner HM, Cox N, Despois D, Ehrenfreund P, Kisiel Z, Lee Y-Y, Markwick AJ, Peeters Z, Rodgers SD (2005) Astronomical searches for nitrogen heterocycles. Adv Space Res 36:137-145

Chiar JE, Tielens AGGM, Whittet DCB, Schutte WA, Boogert ACA, Lutz D, van Dishoeck EF, Bernstein MP (2000) The composition and distribution of dust along the line of sight toward the galactic center. Astrophys J 537:749-762

Chyba C, Sagan C (1992) Endogenous production, exogenous delivery and impact-shock synthesis of organic molecules: an inventory for the origins of life. Nature 355:125-132

Clemett SJ, Maechling CR, Zare RN, Swan PD, Walker RM (1993) Identification of complex aromatic molecules in individual interplanetary dust particles. Science 262:721-725

Clemett SJ, Sandford SA, Nakamura-Messenger K, Hrz F, McKay DS (2010) Complex aromatic hydrocarbons in stardust samples collected from comet 81P/Wild 2. Meteorit Planet Sci 45:701-722

Cody GD, Heying E, Alexander CMO, Nittler LR, Kilcoyne ALD, Sandford SA, Stroud RM (2011) Establishing a molecular relationship between chrondritic and cometary organic solids. Proc Natl Acad Sci USA 108:19171-19176

Colangeli L, Henning T, Brucato JR, Clment D, Fabian D, Guillois O, Huisken F, Jger C, Jessberger EK, Jones A, Ledoux G, Manic G, Mennella V, Molster FJ, Mutschke H, Pirronello V, Reynaud C, Roser J, Vidali G, Waters LBFM (2003) The role of laboratory experiments in the characterisation of silicon-based cosmic material. A A Rev 11(2-3):97-152

Cordiner MA, Nixon C, Charnley SB, Teanby N, Irwin P, Serigano J, Mumma MJ, Palmer M, Kisiel Z, Kuan Y-J, Chuang Y-L (2015) Probing titan's complex atmospheric chemistry using the atacama large millimeter/submillimeter array. IAU Gen Assembl 22:55483

Cruikshank DP, Imanaka H, Dalle Ore CM (2005) Tholins as coloring agents on outer solar system bodies. Adv Space Res 36:178-183

Crockett NR, Bergin EA, Neill JL, Favre C, Schilke P, Lis DC, Bell TA, Blake G, Cernicharo J, Emprechtinger M, Esplugues GB, Gupta H, Kleshcheva M, Lord S, Marcelino N, McGuire BA, Pearson J, Phillips TG, Plume R, van der Tak F, Tercero B, Yu S (2014) Herschel observations of extraordinary sources: analysis of the HIFI 1.2 THz wide spectral survey toward Orion KL. I. Methods. Astrophys J 787:112

Cunningham MR, Jones PA, Godfrey PD, Cragg DM, Bains I, Burton MG, Calisse P, Crighton NHM, Curran SJ, Davis TM, Dempsey JT, Fulton B, Hidas MG, Hill T, Kedziora-Chudczer L, Minier V, Pracy MB, Purcell C, Shobbrook J, Travouillon T (2007) A search for propylene oxide and glycine in Sagittarius B2 (LMH) and Orion. Mon Not R Astron Soc 376:1201-1210

Dartois E, Muoz Caro GM, Deboffle D, d'Hendecourt L (2004) Diffuse interstellar medium organic polymers. Photoproduction of the 3.4, 6.85 and $7.25 \mu \mathrm{m}$ features. Astron Astrophys 423:L33-L36 
Dartois E, Geballe TR, Pino T, Cao A-T, Jones A, Deboffle D, Guerrini V, Brchignac P, D’Hendecourt L (2007) IRAS 08572+3915: constraining the aromatic versus aliphatic content of interstellar HACs. Astron Astrophys 463:635-640

Dartois E (2011) Observations of interstellar carbon compounds. Paper presented at the PAHs and the Universe, March 1, 2011

Dartois E, Engrand C, Brunetto R, Duprat J, Pino T, Quirico E, Remusat L, Bardin N, Briani G, Mostefaoui S, Morinaud G, Crane B, Szwec N, Delauche L, Jamme F, Sandt C, Dumas P (2013) UltraCarbonaceous Antarctic micrometeorites, probing the Solar System beyond the nitrogen snow-line. Icarus 224:243252

Derenne S, Robert F (2010) Model of molecular structure of the insoluble organic matter isolated from Murchison meteorite. Meteorit Planet Sci 45:1461-1475

Díaz-Luis JJ, García-Hernández DA, Kameswara Rao N, Manchado A, Cataldo F (2015) A search for diffuse bands in fullerene planetary nebulae: evidence of diffuse circumstellar bands. Astron Astrophys 573:A97

Dickens JE, Irvine WM, Nummelin A, Mllendal H, Saito S, Thorwirth S, Hjalmarson Ohishi M (2001) Searches for new interstellar molecules, including a tentative detection of aziridine and a possible detection of propenal. Spectrochimica Acta Part A Mol Spectrosc 57:643-660

Dischler B, Bubenzer A, Koidl P (1983) Bonding in hydrogenated hard carbon studied by optical spectroscopy. Solid State Commun 48(2):105-108

Dow WG (1977) Kerogen studies and geological interpretations. J Geochem Explor 7:79-99

Duley WW, Williams DA (1981) The infrared spectrum of interstellar dust-surface functional groups on carbon. Mon Not R Astron Soc 196:269-274

Ehrenfreund P, Cami J, Jimnez-Vicente J, Foing BH, Kaper L, van der Meer A, Cox N, D’Hendecourt L, Maier JP, Salama F, Sarre PJ, Snow TP, Sonnentrucker P (2002a) Detection of diffuse interstellar bands in the magellanic clouds. Astrophys J 576:L117-L120

Ehrenfreund P, Irvine W, Becker L, Blank J, Brucato JR, Colangeli L, Derenne S, Despois D, Dutrey A, Fraaije H, Lazcano A, Owen T, Robert F (2002b) Astrophysical and astrochemical insights into the origin of life. Rep Prog Phys 65(10):1427-1487

Elíasdóttir Á, Fynbo JPU, Hjorth J, Ledoux C, Watson DJ, Andersen AC, Malesani D, Vreeswijk PM, Prochaska JX, Sollerman J, Jaunsen AO (2009) Dust extinction in high-z galaxies with gamma-ray burst afterglow spectroscopy: the 2175 feature at $z=2.45$. Astrophys J 697:1725-1740

Elsila JE, Glavin DP, Dworkin JP (2009) Cometary glycine detected in samples returned by Stardust. Meteorit Planet Sci 44:1323-1330

Falkowski P, Scholes RJ, Boyle E, Canadell J, Canfield D, Elser J, Gruber N, Hibbard K, Hgberg P, Linder S, Mackenzie FT, Moore B, Pedersen T, Rosenthal Y, Seitzinger S, Smetacek V, Steffen W (2000) The global carbon cycle: a test of our knowledge of earth as a system. Science 290:291-296

Feser M, Wirick S, Flynn GJ, Keller LP, Stansbery E (2003) Combined carbon, nitrogen, and oxygen XANES spectroscopy on hydratedand anhydrous interplanetary dust particles. Paper presented at the lunar and planetary science conference, March 1, 2003

Flynn GJ, Keller LP, Feser M, Wirick S, Jacobsen C (2003) The origin of organic matter in the solar system: evidence from the interplanetary dust particles. Geochimica et Cosmochimica Acta 67:4791-4806

Foing BH, Ehrenfreund P (1994) Detection of two interstellar absorption bands coincident with spectral features of $\mathrm{C}_{60}^{+}$. Nature 369:296-298

Forrest WJ, Houck JR, McCarthy JF (1981) A far-infrared emission feature in carbon-rich stars and planetary nebulae. Astrophys J 248:195-200

Fourikis N, Takagi K, Morimoto M (1974) Detection of interstellar methylamine by its $2_{02}-1_{10} A_{a}$ - state transition. Astrophys J Lett 191:L139

García-Hernández DA, Díaz-Luis JJ (2013) Diffuse interstellar bands in fullerene planetary nebulae: the fullerenes - diffuse interstellar bands connection. Astron Astrophys 550:L6

García-Hernández DA, Villaver E, García-Lario P, Acosta-Pulido JA, Manchado A, Stanghellini L, Shaw RA, Cataldo F (2012) Infrared study of fullerene planetary nebulae. Astrophys J 760:107

Geballe TR, Tielens AGGM, Kwok S, Hrivnak BJ (1992) Unusual 3 micron emission features in three proto-planetary nebulae. Astrophys J 387:L89-L91

Giri C, McKay CP, Goesmann F, Schfer N, Li X et al (2015) Carbonization in Titan Tholins: implication for low albedo on surfaces of Centaurs and trans-Neptunian objects. Int J Astrobiol (in press)

Goesmann F, Rosenbauer H, Bredehft JH, Cabane M, Ehrenfreund P, Gautier T, Giri C, Krger H, Le Roy L, MacDermott AJ, McKenna-Lawlor S, Meierhenrich UJ, Caro GMM, Raulin F, Roll R, Steele 
A, Steininger H, Sternberg R, Szopa C, Thiemann W, Ulamec S (2015) Organic compounds on comet67P/Churyumov-Gerasimenko revealed by COSAC mass spectrometry. Science 349(6247)

Gradie J, Veverka J (1980) The composition of the Trojan asteroids. Nature 283:840-842

Gredel R, Carpentier Y, Rouill G, Steglich M, Huisken F, Henning T (2011) Abundances of PAHs in the ISM: confronting observations with experimental results. Astron Astrophys 530:26

Guillois O, Nenner I, Papoular R, Reynaud C (1996) Coal models for the infrared emission spectra of proto-planetary nebulae. Astrophys J 464:810-817

Halfen DT, Ilyushin V, Ziurys LM (2011) Formation of peptide bonds in space: a comprehensive study of formamide and acetamide in Sgr B2(N). Astrophys J 743:60

Heger ML (1922) Lick Observ Bull 10:146

Herlin N, Bohn I, Reynaud C, Cauchetier M, Galvez A, Rouzaud J-N (1998) Nanoparticles produced by laser pyrolysis of hydrocarbons: analogy with carbon cosmic dust. Astron Astrophys 330:1127-1135

Hollis JM, Lovas FJ, Jewell PR (2000) Interstellar glycolaldehyde: the first sugar. Astrophys J 540:L107L110

Hollis JM, Jewell PR, Lovas FJ, Remijan A, Møllendal H (2004) Green bank telescope detection of new interstellar aldehydes: propenal and propanal. Astrophys J 610:L21-L24

Hollis JM, Lovas FJ, Remijan AJ, Jewell PR, Ilyushin VV, Kleiner I (2006) Detection of acetamide $(\mathrm{CH} 3 \mathrm{CONH} 2)$ : the largest interstellar molecule with a peptide bond. Astrophys J 643:L25-L28

Hoyle F, Wickramasinghe NC (1977) Polysaccharides and infrared spectra of galactic sources. Nature 268:610-612

Hrivnak BJ, Volk K, Kwok S (2000) 2-45 micron infrared spectroscopy of carbon-rich proto-planetary nebulae. Astrophys J 535:275-292

Hu A, Duley WW (2008) Spectra of carbon nanoparticles: laboratory simulation of the aromatic CH emission feature at 3.29 $\mu \mathrm{m}$. Astrophys J Lett 677:L153-L156

Iglesias-Groth S (2004) Fullerenes and buckyonions in the interstellar medium. Astrophys J Lett 608:L37L40

Imanishi M, Nakagawa T, Shirahata M, Ohyama Y, Onaka T (2010) AKARI IRC infrared 2.5-5 m spectroscopy of a large sample of luminous infrared galaxies. Astrophys J 721:1233-1261

Irvine WM, Ellder J, Hjalmarson A, Kollberg E, Rydbeck OEH, Sorensen GO, Bak B, Svanholt H (1981) Searches for interstellar imidazole and cyanoform. Astron Astrophys 97:192-194

Jäger C, Huisken F, Mutschke H, Jansa IL, Henning TH (2009) Formation of polycyclic aromatic hydrocarbons and carbonaceous solids in gas-phase condensation experiments. Astrophys J 696(1):706-712. doi: $10.1088 / 0004-637 x / 696 / 1 / 706$

Jones AP (2012a) Variations on a theme-the evolution of hydrocarbon solids. I. Compositional and spectral modelling - the eRCN and DG models. Astron Astrophys 540:1

Jones AP (2012b) Variations on a theme-the evolution of hydrocarbon solids. II. Optical property modelling - the optEC(s) model. Astron Astrophys 540:2

Jones AP (2012c) Variations on a theme-the evolution of hydrocarbon solids (Corrigendum). III. Sizedependent properties - the optEC(s)(a) model. Astron Astrophys 545:3

Jones AP, Fanciullo L, Khler M, Verstraete L, Guillet V, Bocchio M, Ysard N (2013) The evolution of amorphous hydrocarbons in the ISM: dust modelling from a new vantage point. Astron Astrophys 558:62

Jones PA, Cunningham MR, Godfrey PD, Cragg DM (2007) A search for biomolecules in sagittarius B2 (LMH) with the Australia telescope compact array. Mon Not R Astron Soc 374:579-589

Jørgensen JK, Favre C, Bisschop SE, Bourke TL, van Dishoeck EF, Schmalzl M (2012) Detection of the simplest sugar, glycolaldehyde, in a solar-type protostar with ALMA. Astrophys J Lett 757:L4

Jourdain de Muizon M, D’Hendecourt LB, Geballe TR (1990) Three micron spectroscopy of IRAS sources-observed and laboratory signatures of PAHs. Astron Astrophys 235:367-378

Kahanpää J, Mattila K, Lehtinen K, Leinert C, Lemke D (2003) Unidentified infrared bands in the interstellar medium across the Galaxy. Astron Astrophys 405:999-1012

Kahane C, Ceccarelli C, Faure A, Caux E, Kuno N, Yamamoto S (2013) Detection of Formamide in the Solar-Type Protostar IRAS16293-2422. Paper presented at the new trends in radio astronomy in the ALMA era: the 30th anniversary of Nobeyama radio observatory, vol 1, 2013

Kaifu N, Morimoto M, Nagane K, Akabane K, Iguchi T, Takagi K (1974) Detection of interstellar methylamine. Astrophys J Lett 191:L135-L137

Keller LP, Bajt S, Baratta GA, Borg J, Bradley JP, Brownlee DE, Busemann H, Brucato JR, Burchell M, Colangeli L, D’Hendecourt L, Djouadi Z, Ferrini G, Flynn G, Franchi IA, Fries M, Grady MM, 
Graham GA, Grossemy F, Kearsley A, Matrajt G, Nakamura-Messenger K, Mennella V, Nittler L, Palumbo ME, Stadermann FJ, Tsou P, Rotundi A, Sandford SA, Snead C, Steele A, Wooden D, Zolensky M (2006) Infrared spectroscopy of comet 81P/Wild 2 samples returned by Stardust. Science 314:1728-1731

Kerridge JF (1999) Formation and processing of organics in the early solar system. Space Sci Rev 90:275288

Kim YS, Kaiser RI (2011) On the formation of amines $\left(\mathrm{RNH}_{2}\right)$ and the cyanide anion $\left(\mathrm{CN}^{-}\right)$in electronirradiated ammonia-hydrocarbon interstellar model ices. Astrophys J 729:68

Kim SJ, Jung A, Sim CK, Courtin R, Bellucci A, Sicardy B, Song IO, Minh YC (2011) Retrieval and tentative identification of the $3 \mu \mathrm{m}$ spectral feature in Titan's haze. Planet Space Sci 59:699-704

Kim SJ, Sim CK, Lee DW, Courtin R, Moses JI, Minh YC (2012) The three-micron spectral feature of the Saturnian haze: implications for the haze composition and formation process. Planet Space Sci 65:122-129

Knacke RF (1977) Carbonaceous compounds in interstellar dust. Nature 269:132-134

Kroto HW, Heath JR, Obrien SC, Curl RF, Smalley RE (1985) C(60): Buckminsterfullerene. Nature 318:162

Kuan YJ, Charnley SB, Huang HC, Tseng WL, Kisiel Z (2003a) Interstellar glycine. Astrophys J 593(2):848-867

Kuan Y-J, Yan C-H, Charnley SB, Kisiel Z, Ehrenfreund P, Huang H-C (2003b) A search for interstellar pyrimidine. Mon Not R Astron Soc 345:650-656

Kutner ML, Machnik DE, Tucker KD, Dickman RL (1980) Search for interstellar pyrrole and furan. Astrophys J 242:541-544

Kwok S (2004) The synthesis of organic and inorganic compounds in evolved stars. Nature 430:985-991

Kwok S (2006) Physics and chemistry of the interstellar medium. University Science Books, USA

Kwok S (2014) Organic nanoparticles as a component of the interstellar medium. IAU symposium 297: the diffuse interstellar bands, CUP, p 213

Kwok S, Zhang Y (2011) Mixed aromatic-aliphatic organic nanoparticles as carriers of unidentified infrared emission features. Nature 479:80-83

Kwok S, Zhang Y (2013) Unidentified infrared emission bands: PAHs or MAONs? Astrophys J 771:5

Kwok S, Volk KM, Hrivnak BJ (1989) A 21 micron emission feature in four proto-planetary nebulae. Astrophys J 345:L51-L54

Kwok S, Volk K, Bernath P (2001) On the origin of infrared plateau features in proto-planetary nebulae. Astrophys J 554:L87-L90

Ledoux G, Ehbrecht M, Guillois O, Huisken F, Kohn B, Laguna MA, Nenner I, Paillard V, Papoular R, Porterat D, Reynaud C (1998) Silicon as a candidate carrier for ERE. Astron Astrophys 333:L39-L42

Léger A, d'Hendecourt L (1985) Are polycyclic aromatic hydrocarbons the carriers of the diffuse interstellar bands in the visible? Astron Astrophys 146:81-85

Léger A, Puget JL (1984) Identification of the 'unidentified' IR emission features of interstellar dust? Astron Astrophys 137:L5-L8

Licandro J, Campins H, Kelley M, Hargrove K, Pinilla-Alonso N, Cruikshank D, Rivkin AS, Emery J (2011) (65) Cybele: detection of small silicate grains, water-ice, and organics. Astron Astrophys 525:34

Lorenz RD, Mitchell KL, Kirk RL, Hayes AG, Aharonson O, Zebker HA, Paillou P, Radebaugh J, Lunine JI, Janssen MA, Wall SD, Lopes RM, Stiles B, Ostro S, Mitri G, Stofan ER (2008) Titan's inventory of organic surface materials. Geophys Res Lett 35:02206

Lutz D, Sturm E, Tacconi LJ, Valiante E, Schweitzer M, Netzer H, Maiolino R, Andreani P, Shemmer O, Veilleux S (2007) PAH emission and star formation in the host of the $z \sim 2.56$ cloverleaf QSO. Astrophys J Lett 661:L25-L28

Mason RE, Wright G, Pendleton Y, Adamson A (2004) Hydrocarbon dust absorption in seyfert galaxies and ultraluminous infrared galaxies. Astrophys J 613:770-780

Materese CK, Cruikshank DP, Sandford SA, Imanaka H, Nuevo M, White DW (2014) Ice chemistry on outer solar system bodies: carboxylic acids, nitriles, and urea detected in refractory residues produced from the UV photolysis of $\mathrm{N}_{2}: \mathrm{CH}_{4}$ :CO-containing ices. Astrophys $\mathrm{J} 788: 111$

Matrajt G, Flynn G, Brownlee D, Joswiak D, Bajt S (2013) The origin of the $3.4 \mathrm{~m}$ feature in wild 2 cometary particles and in ultracarbonaceous interplanetary dust particles. Astrophys J 765:145

McKellar A (1940) Evidence for the molecular origin of some hitherto unidentified interstellar lines. Publ Astron Soc Pac 52:187 
Mendoza E, Lefloch B, Lpez-Sepulcre A, Ceccarelli C, Codella C, Boechat-Roberty HM, Bachiller R (2014) Molecules with a peptide link in protostellar shocks: a comprehensive study of L1157. Mon Not R Astron Soc 445:151-161

Mennella V, Colangeli L, Bussoletti E, Palumbo P, Rotundi A (1998) A new approach to the puzzle of the ultraviolet interstellar extinction bump. Astrophys J Lett 507:L177-L180

Mennella V, Brucato JR, Colangeli L, Palumbo P (1999) Activation of the 3.4 micron band in carbon grains by exposure to atomic hydrogen. Astrophys J 524:L71-L74

Mennella V, Baratta GA, Esposito A, Ferini G, Pendleton YJ (2003) The effects of ion irradiation on the evolution of the carrier of the 3.4 micron interstellar absorption band. Astrophys J 587:727-738

Messenger SR, Nakamura-Messenger K (2015) Interstellar and solar system organic matter preserved in interplanetary dust. IAU Gen Assembl 22:59062

Mumma MJ, Charnley SB (2011) The chemical composition of comets-emerging taxonomies and natal heritage. Annu Rev Astron Astrophys 49:471-524

Nagy B, Meinschein WG, Hennessy DJ (1961) Mass spectroscopic analysis of the Orgueil meteorite: evidence for biogenic hydrocarbons. Ann NY Acad Sci 93(2):27-35

Naraoka H, Aoki D, Fukushima K, Uesugi M, Ito M, Kitajima F, Mita H, Yabuta H, Takano Y, Yada T, Ishibashi Y, Karouji Y, Okada T, Abe M (2015) ToF-SIMS analysis of carbonaceous particles in the sample catcher of the Hayabusa spacecraft. Earth Planets Space 67:67

Neill JL, Bergin EA, Lis DC, Schilke P, Crockett NR, Favre C, Emprechtinger M, Comito C, Qin S-L, Anderson DE, Burkhardt AM, Chen J-H, Harris BJ, Lord SD, McGuire BA, McNeill TD, Monje RR, Phillips TG, Steber AL, Vasyunina T, Yu S (2014) Herschel observations of extraordinary sources: analysis of the full Herschel/HIFI molecular line survey of Sagittarius B2(N). Astrophys J 789:8

Ohishi M (2015) Private communication

Otsuka M, Kemper F, Hyung S, Sargent BA, Meixner M, Tajitsu A, Yanagisawa K (2013) The detection of $\mathrm{C}_{60}$ in the well-characterized planetary nebula M1-11. Astrophys J 764:77

Papoular R (2001) The use of kerogen data in understanding the properties and evolution of interstellar carbonaceous dust. Astron Astrophys 378:597-607

Papoular R (2011) Candidate carriers and synthetic spectra of the 21- and 30-m proto-planetary nebular bands. Mon Not R Astron Soc 415:494-502

Papoular RJ, Papoular R (2009) A polycrystalline graphite model for the 2175 interstellar extinction band. Mon Not R Astron Soc 394:2175-2181

Papoular R, Conrad J, Giuliano M, Kister J, Mille G (1989) A coal model for the carriers of the unidentified IR bands. Astron Astrophys 217:204-208

Pasek MA (2016) Implications of extraterrestrial material on the origin of life. In: Benvenuti P (ed) Astronomy in focus, vol 1 (in press)

Pendleton YJ, Sandford SA, Allamandola LJ, Tielens AGGM, Sellgren K (1994) Near-infrared absorption spectroscopy of interstellar hydrocarbon grains. Astrophys J 437:683-696

Pino T, Dartois E, Cao A-T, Carpentier Y, Chamaill T, Vasquez R, chignac P (2008) The 6.2 m band position in laboratory and astrophysical spectra: a tracer of the aliphatic to aromatic evolution of interstellar carbonaceous dust. Astron Astrophys 490:665-672

Pizzarello S, Cronin JR (2000) Non-racemic amino acids in the Murray and Murchison meteorites. Geochimica Et Cosmochimica Acta 64:329-338

Pizzarello S, Shock E (2010) The organic composition of carbonaceous meteorites: the evolutionary story ahead of biochemistry. Cold Spring Harbor Perspect Biol 2:a002105

Pope A, Wagg J, Frayer D, Armus L, Chary R-R, Daddi E, Desai V, Dickinson ME, Elbaz D, Gabor J, Kirkpatrick A (2013) Probing the interstellar medium of $z \sim 1$ ultraluminous infrared galaxies through interferometric observations of CO and spitzer mid-infrared spectroscopy. Astrophys J 772:92

Puetter RC, Russell RW, Willner SP, Soifer BT (1979) Spectrophotometry of compact H II regions from 4 to 8 microns. Astrophys J 228:118-122

Remijan AJ, Snyder LE, McGuire BA, Kuo H-L, Looney LW, Friedel DN, Golubiatnikov GY, Lovas FJ, Ilyushin VV, Alekseev EA, Dyubko SF, McCall BJ, Hollis JM (2014) Observational results of a multi-telescope campaign in search of interstellar urea [(NH2) $\left.{ }_{2} \mathrm{CO}\right]$. Astrophys J 783:77

Risaliti G, Maiolino R, Marconi A, Sani E, Berta S, Braito V, Della Ceca R, Franceschini A, Salvati M (2006) Unveiling the nature of ultraluminous infrared galaxies with 3-4m spectroscopy*. Mon Not R Astron Soc 365:303-320

Robertson J (2002) Diamond-like amorphous carbon. Mater Sci Eng R Rep 37(4-6):129-281 
Rubin RH, Swenson GW Jr, Benson RC, Tigelaar HL, Flygare WH (1971) Microwave detection of interstellar formamide. Astrophys J 169:L39

Russell RW, Soifer BT, Willner SP (1977) The 4 to 8 micron spectrum of NGC 7027. Astrophys J 217:L149L153

Sadjadi S, Zhang Y, Kwok S (2015) On the origin of the 11.3 micron unidentified infrared emission feature. Astrophys J 807:95

Sagan C, Khare BN (1979) Tholins-organic chemistry of interstellar grains and gas. Nature 277:102-107

Sakata A, Wada S, Onaka T, Tokunaga AT (1987) Infrared spectrum of quenched carbonaceous composite (QCC). II-a new identification of the 7.7 and 8.6 micron unidentified infrared emission bands. Astrophys J 320:L63-L67

Sakata A, Wada S, Narisawa T, Asano Y, Iijima Y, Onaka T, Tokunaga AT (1992) Quenched carbonaceous composite-fluorescence spectrum compared to the extended red emission observed in reflection nebulae. Astrophys J 393:L83-L86

Salama F, Ehrenfreund P (2014) A critical review of PAHs as DIB carriers-progress and open questions. In: Cami J, Cox N (eds) IAU symposium 297: the diffuse interstellar bands, CUP, p 364

Salama F, Galazutdinov GA, Krelowski J, Biennier L, Beletsky Y, Song I-O (2011) Polycyclic aromatic hydrocarbons and the diffuse interstellar bands: a survey. Astrophys J 728:154

Sarre PJ (2006) The diffuse interstellar bands: a major problem in astronomical spectroscopy. J Mol Spectrosc 238(1):1-10

Schmidt GD, Witt AN (1991) X marks the SPOT_-distribution and excitation of unidentified molecules in the red rectangle. Astrophys J 383:698-704

Schmitt-Kopplin P, Gabelica Z, Gougeon RD, Fekete A, Kanawati B, Harir M, Gebefuegi I, Eckel G, Hertkorn N (2010) High molecular diversity of extraterrestrial organic matter in Murchison meteorite revealed 40 years after its fall. Proc Natl Acad Sci 107:2763-2768

Scott A, Duley WW (1996) The decomposition of hydrogenated amorphous carbon: a connection with polycyclic aromatic hydrocarbon molecules. Astrophys J 472:L123

Sephton MA, Love GD, Watson JS, Verchovsky AB, Wright IP, Snape CE, Gilmour I (2004) Hydropyrolysis of insoluble carbonaceous matter in the Murchison meteorite: new insights into its macromolecular structure1. Geochimica et Cosmochimica Acta 68(6):1385-1393

Smith JDT, Draine BT, Dale DA, Moustakas J, Kennicutt RC Jr, Helou G, Armus L, Roussel H, Sheth K, Bendo GJ, Buckalew BA, Calzetti D, Engelbracht CW, Gordon KD, Hollenbach DJ, Li A, Malhotra S, Murphy EJ, Walter F (2007) The mid-infrared spectrum of star-forming galaxies: global properties of polycyclic aromatic hydrocarbon emission. Astrophys J 656:770-791

Snow TP (2014) Diffuse interstellar bands: past and present. In: Cami J, Cox NLJ (eds) IAU symposium 297: the interstellar diffuse bands, CUP, p 3

Snyder LE, Lovas FJ, Hollis JM, Friedel DN, Jewell PR, Remijan A, Ilyushin VV, Alekseev EA, Dyubko SF (2005) A rigorous attempt to verify interstellar glycine. Astrophys J 619:914-930

Sollerman J, Cox N, Mattila S, Ehrenfreund P, Kaper L, Leibundgut B, Lundqvist P (2005) Diffuse interstellar bands in NGC 1448. Astron Astrophys 429:559-567

Speck AK, Hofmeister AM (2004) Processing of presolar grains around post-asymptotic giant branch stars: silicon carbide as the carrier of the 21 micron feature. Astrophys J 600(2):986-991

Stecher TP (1965) Interstellar extinction in the ultraviolet. Astrophys J 142:1683

Teplitz HI, Desai V, Armus L, Chary R, Marshall JA, Colbert JW, Frayer DT, Pope A, Blain A, Spoon HWW, Charmandaris V, Scott D (2007) Measuring PAH emission in ultradeep spitzer IRS spectroscopy of high-redshift IR-luminous galaxies. Astrophys J 659:941-949

Townes CH (1957) Micrwave and radio frequency resonance lines of interest to radio astronomy. In: van de Hulst (ed) IAU Symp. 4: radio astronomy, CUP, p 92

Townes CH (2006) The discovery of interstellar water vapor and ammonia at the Hat Creek Radio Observatory. In: Backer, Turner, Moran (eds) Revealing the molecular universe, ASP Conf. Ser. 356, p 81

Turner BE, Liszt HS, Kaifu N, Kisliakov AG (1975) Microwave detection of interstellar cyanamide. Astrophys J Lett 201:L149-L152

Valiante E, Lutz D, Sturm E, Genzel R, Tacconi LJ, Lehnert MD, Baker AJ (2007) A mid-infrared spectroscopic study of submillimeter galaxies: luminous starbursts at high redshift. Astrophys $\mathrm{J}$ 660:1060-1071 
Van Kerckhoven C, Hony S, Peeters E, Tielens A, Allamandola LJ, Hudgins DM, Cox P, Roelfsema PR, Voors RHM, Waelkens C, Waters L, Wesselius PR (2000) The C-C-C bending modes of PAHs: a new emission plateau from 15 to $20 \mu \mathrm{m}$. Astron Astrophys 357(3):1013-1019

Van Winckel H, Cohen M, Gull TR (2002) The ERE of the "Red Rectangle" revisited. Astron Astrophys 390:147-154

Vijh UP, Witt AN, Gordon KD (2004) Discovery of blue luminescence in the red rectangle: possible fluorescence from neutral polycyclic aromatic hydrocarbon molecules? Astrophys J 606:L65-L68

Volk K, Kwok S, Hrivnak BJ (1999) High-resolution infrared space observatory spectroscopy of the unidentified 21 micron feature. Astrophys J 516:L99-L102

Volk K, Hrivnak BJ, Matsuura M, Bernard-Salas J, Szczerba R, Sloan GC, Kraemer KE, van Loon JT, Kemper F, Woods PM, Zijlstra AA, Sahai R, Meixner M, Gordon KD, Gruendl RA, Tielens AGGM, Indebetouw R, Marengo M (2011) Discovery and analysis of $21 \mu \mathrm{m}$ feature sources in the magellanic clouds. Astrophys J 735:127

Walker GAH, Bohlender DA, Maier JP, Campbell EK (2015) Astrophys J Lett 812:L8

Webster A (1993) The extended red emission and the fluorescence of C60. Mon Not R Astron Soc 264:L1

Webster A (1995) The lowest of the strongly infrared active vibrations of the fulleranes and astronomical emission band at a wavelength of 21-microns. Royal Astron Soc Monthly Notices 277(4):1555

Westphal AJ et al (2014) Evidence for interstellar origin of seven dust particles collected by the Stardust spacecraft. Science 345:786-791

Wickramasinghe DT, Allen DA (1980) The 3.4-micron interstellar absorption feature. Nature 287:518

Winn JN, Fabrycky DC (2015) The occurrence and architecture of exoplanetary systems. Annu Rev Astron Astrophys 53(1):409-447. doi:10.1146/annurev-astro-082214-122246

Witt AN, Boroson TA (1990) Spectroscopy of extended red emission in reflection nebulae. Astrophys J 355:182-189

Witt AN, Schild RE (1988) Hydrogenated amorphous carbon grains in reflection nebulae. Astrophys J 325:837-845

Witt AN, Gordon KD, Furton DG (1998) Silicon nanoparticles: source of extended red emission? Astrophys J 501:L111

Woolf NJ, Ney EP (1969) Circumstellar Infrared emission from cool stars. Astrophys J 155:L181

Zhang Y, Kwok S (2011) Detection of $\mathrm{C}_{60}$ in the protoplanetary nebula IRAS 01005+7910. Astrophys J 730:126

Zhang Y, Kwok S (2013) On the detections of $\mathrm{C}_{60}$ and derivatives in circumstellar environments. Earth Planets Space 65:1069-1081

Zhang Y, Kwok S (2015) On the viability of the PAH model as an explanation of the unidentified infrared emission features. Astrophys J 798:37

Zhang Y, Kwok S, Hrivnak BJ (2010) A spitzer/infrared spectrograph spectral study of a sample of galactic carbon-rich proto-planetary nebulae. Astrophys J 725:990-1001

Zhang K, Jiang BW, Li A (2009) On magnesium sulfide as the carrier of the $30 \mu \mathrm{m}$ emission feature in evolved stars. Astrophys J 702:680-685 